Document downloaded from:

http://hdl.handle.net/10251/146171

This paper must be cited as:

Kumar, A.; Gupta, D.; Martínez Molada, E.; Singh, S. (04-2). Directional k-Step Newton Methods in $n$ Variables and its Semilocal Convergence Analysis. Mediterranean Journal of Mathematics. 15(2):15-34. https://doi.org/10.1007/s00009-018-1077-0

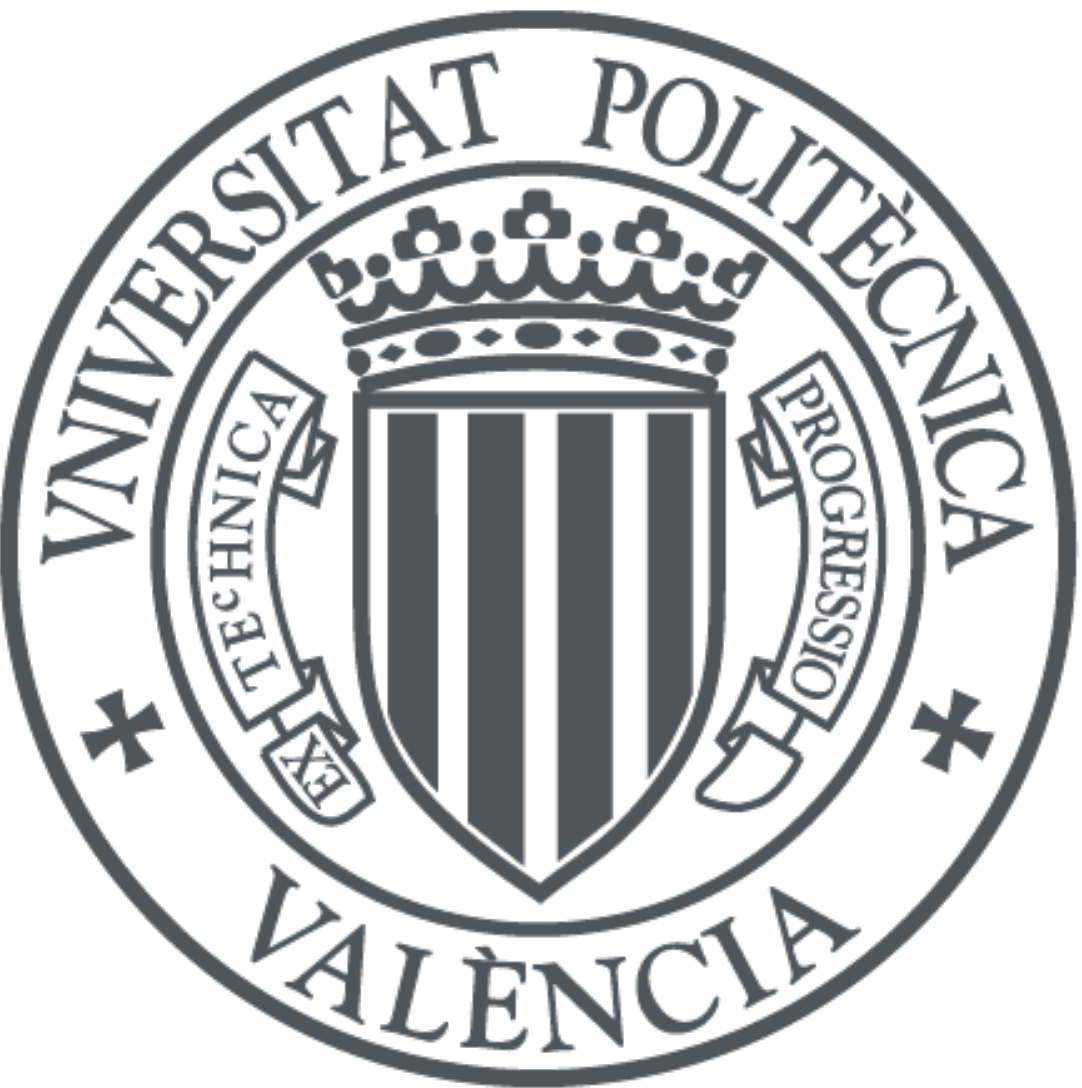

The final publication is available at

https://doi.org/10.1007/s00009-018-1077-0

Copyright Springer-Verlag

Additional Information 


\title{
Directional k-step Newton methods in $n$ variables and its semilocal convergence analysis
}

\author{
A. Kumar ${ }^{\text {a }}$, D.K. Gupta ${ }^{\text {a }}$, Eulalia Martinez, ${ }^{\text {b }}$, Sukhjit Singh, ${ }^{\text {c }}$ \\ ${ }^{a}$ Department of Mathematics, Indian Institute of Technology Kharagpur, \\ 721302, India. \\ E-mail address: akr.math01991@gmail.com, dkg@maths.iitkgp.ernet.in \\ ${ }^{\mathrm{b}}$ Instituto de Matemática Pura y Aplicada, Universitat Politècnica de València, \\ 46022 Valencia, Spain. \\ E-mail address: eumarti@mat.upv.es \\ ${ }^{\mathrm{c}}$ Department of Mathematics, National Institute of Technology Hamirpur, \\ 177005, India. \\ E-mail address: sukhjitmath@gmail.com
}

February 21, 2017

\begin{abstract}
The directional k-step Newton methods ( $k$ a positive integer) is developed for solving a single nonlinear equation in $n$ variables. Its semilocal convergence analysis is established by using two different approaches (recurrent relations and recurrent functions) under the assumption that the first derivative satisfies a combination of the Lipschitz and the center-Lipschitz continuity conditions instead of only Lipschitz condition. The convergence theorems for the existence and uniqueness of the solution for each of them are established. Numerical examples including nonlinear Hammerstein type integral equations are worked out and significantly improved results are obtained. It is shown that the second approach based on recurrent functions solves problems failed to be solved by first one using recurrent relations. This demonstrates the efficacy and applicability of these approaches. This work extends the directional one and two-step Newton methods for solving a single nonlinear equation in $n$ variables. Their semilocal convergence analysis using majorizing sequences are studied in [1] and [5] under the assumption that the first derivative satisfies the Lipschitz and the combination of the Lipschitz and the center-Lipschitz continuity conditions, respectively. Finally, the computational order of convergence and the computational efficiency of developed method are studied.
\end{abstract}

Keywords: Directional Newton methods, Nonlinear equations, Recurrent relations, Recurrent functions, Majorizing sequences, Semilocal convergence analysis.

2010 Mathematics Subject Classification: Primary 65H05, 65H10, 49M15. 


\section{Introduction}

This paper deals with the problem of approximating a solution $x^{*}$ of nonlinear equation in $n$ variables

$$
\mathscr{F}(x)=0
$$

where, $\mathscr{F}: \mathscr{D} \subset \mathscr{R}^{n} \rightarrow \mathscr{R}$ is a Fréchet-differentiable mapping defined in an open convex nonempty subset $\mathscr{D}$ of $\mathscr{R}^{n}$ ( $n$ a natural number) with values in $\mathscr{R}$. These problems are solved by mathematical tools involving integral equations, boundary value problems, differential equations whose solutions require solving either scalar equations or system of equations. Many researchers [1, 2, 3, 4, 5, 19] have studied these problems and a large number of diverse numerical methods are proposed for their solutions. The existence of high speed computing devices helps in solving them faster with more accuracy. This and many other related problems appear in several applications of computational and engineering sciences such as economic equilibrium theory, optimization, elasticity and dynamical systems. For example, finding a local minimum of function is connected to solving a set of nonlinear equations. Computer graphics applications involve intersection of two surfaces which become complicated because of some closed loops and singularities $[1,2]$. They require finding efficient algorithms for computing and displaying this intersection. If $\mathscr{S}_{1}$ and $\mathscr{S}_{2}$ are two surfaces in $\mathscr{R}^{3}$ then the solution of their intersection $\mathscr{S}=\mathscr{S}_{1} \cap \mathscr{S}_{2}$ must satisfy the nonlinear equation which is a special case of (1). More precisely, if these surfaces are represented explicitly as $\mathscr{S}_{1}:=\left\{(a, b, c)^{T}: c=g_{1}(a, b)\right\}$ and $\mathscr{S}_{2}:=\left\{(a, b, c)^{T}: c=g_{2}(a, b)\right\}$ then the solution $x^{*}=\left(a^{*}, b^{*}, c^{*}\right)^{T} \in \mathscr{S}$ satisfies the nonlinear equations given by $g_{1}\left(a^{*}, b^{*}\right)=g_{2}\left(a^{*}, b^{*}\right)$ and $c^{*}=g_{1}\left(a^{*}, b^{*}\right)$. This is obtained by solving a nonlinear equation of the form $g(x)=g_{1}(x)-g_{2}(x)=0$, where $x=(a, b)^{T}$. The marching method can also be used to compute the intersection $\mathscr{S}=\mathscr{S}_{1} \cap \mathscr{S}_{2}$. Here, starting with suitable $x^{0}=\left(a^{0}, b^{0}, c^{0}\right)^{T} \in \mathscr{S}$ succeeding intersection points are computed using successive updating. This means that after getting a point $x^{k}=\left(a^{k}, b^{k}, c^{k}\right)^{T} \in \mathscr{S}$ at the k-th step, we need to search the updated point at $(\mathrm{k}+1)$-th step. This can be taken as the most suitable point to $x^{k}$ among three points obtained by solving the nonlinear equation (1), with $x_{j}=x_{j}^{k}+\Delta_{j}^{k}, \Delta_{j}^{k}$ is sufficiently small, $j=1,2,3$. For $x_{j}^{k} \in\left\{a^{k}, b^{k}, c^{k}\right\}, \Delta_{j}^{k}$ gives a movement in the $x_{j}$-direction. In mathematical programming, the convex composite optimization problem [3] given by

$$
\min _{x \in \mathscr{R}^{n}} f(x):=h(G(x))
$$

where, $h: \mathscr{R}^{n} \rightarrow \mathscr{R}$ and $G: \mathscr{R}^{l} \rightarrow \mathscr{R}^{n}$ is convex and Fréchet differentiable operator, respectively is related to finding the solution of $f(x)=0$.

The directional Newton methods $[1,4]$ are also used to solve (1). Here, $\mathscr{F}$ is restricted to the line $\mathscr{L}:=\left\{x^{0}+\gamma d_{0}: \gamma \in \mathscr{R}\right\}$, where it becomes a function of one variable $f(\gamma)=\mathscr{F}\left(x_{0}+\gamma d_{0}\right)$. Starting with a suitably chosen starting point $x_{0} \in \mathscr{D}$ and a direction vector $d_{0}$, the next point of Newton iteration for $f$ at $\gamma_{0}=0$ is obtained by $\gamma_{1}:=-\frac{f(0)}{f^{\prime}(0)}$. The corresponding iteration for $\mathscr{F}$ is

$$
x_{1}=x_{0}-\frac{\mathscr{F}\left(x_{0}\right)}{\nabla \mathscr{F}\left(x_{0}\right) \cdot d_{0}} d_{0} .
$$

It is also to be noted that $f^{\prime}(0)=\mathscr{F}^{\prime}\left(x_{0}, d_{0}\right)=\nabla \mathscr{F}\left(x_{0}\right) \cdot d_{0} \in \mathscr{R}$. The DNM is given for $n \geq 0$, by

$$
x_{n+1}=x_{n}-\frac{\mathscr{F}\left(x_{n}\right)}{\nabla \mathscr{F}\left(x_{n}\right) \cdot d_{n}} d_{n},
$$

where, $\nabla \mathscr{F}\left(x_{n}\right)=\left(\frac{\partial \mathscr{F}\left(x_{n}\right)}{\partial x_{1}}, \ldots, \frac{\partial \mathscr{F}\left(x_{n}\right)}{\partial x_{n}}\right)$ is the gradient of $\mathscr{F}$ and $d_{n}$ is the directional vector at $x_{n}$. The convergence of (2) was established for directions $d_{n}$ sufficiently close to the gradients $\nabla \mathscr{F}\left(x_{n}\right)$, and under 
standard Newton-Kantorovich-type sufficient conditions [18]. Two well-known approaches for the convergence analysis of iterative methods are local [8, 10, 13], and semilocal convergence [12, 14] analysis. The local and semilocal convergence analysis use information on the solution and on the initial starting approximation to the solution, respectively. Theorems are established to give sufficient conditions for the existence and uniqueness and the radii of convergence balls of the solutions. In [1], the semilocal convergence analysis of (2) is established under the assumption that the first derivative satisfies the stronger Lipschitz continuity condition. Argyros [4] established the semilocal convergence analysis of (2) using a combination of Lipschitz and center-Lipschitz continuity condition on the derivative. This gave weaker convergence conditions, larger convergence domain, finer error estimates on the distances involved and at least a precise information on the location of the solution of the function. In [5], the directional two-step Newton methods and its semilocal convergence analysis for (1) was presented using a combination of Lipschitz and center-Lipschitz continuity condition on the first Fréchet derivative in Hilbert space setting by using recurrent functions and recurrent sequences. It is given for $n \geq 0$, by

$$
\begin{aligned}
y_{n} & =x_{n}-\frac{\mathscr{F}\left(x_{n}\right)}{\nabla \mathscr{F}\left(x_{n}\right) \cdot d_{n}} d_{n} \\
x_{n+1} & =y_{n}-\frac{\mathscr{F}\left(y_{n}\right)}{\nabla \mathscr{F}\left(x_{n}\right) \cdot d_{n}} d_{n},
\end{aligned}
$$

where, $x_{0} \in \mathscr{D}$ and $d_{0}$ are suitably chosen starting point and direction vector, respectively.

Motivated from the work in [5], a directional k-step Newton methods which generalizes (2) and (3)for solving (1) is developed. It is given for $n \geq 0$, by

$$
\begin{aligned}
& x_{n}^{1}=x_{n}^{0}-\frac{\mathscr{F}\left(x_{n}^{0}\right)}{\nabla \mathscr{F}\left(x_{n}^{0}\right) \cdot d_{n}} d_{n} \\
& x_{n}^{2}=x_{n}^{1}-\frac{\mathscr{F}\left(x_{n}^{1}\right)}{\nabla \mathscr{F}\left(x_{n}^{0}\right) \cdot d_{n}} d_{n} \\
& x_{n}^{k}=x_{n}^{k-1}-\frac{\mathscr{F}\left(x_{n}^{k-1}\right)}{\nabla \mathscr{F}\left(x_{n}^{0}\right) \cdot d_{n}} d_{n}
\end{aligned}
$$

where $x_{0}^{0}$ is a starting point, $x_{n+1}^{0}=x_{n}^{k}, \nabla \mathscr{F}\left(x_{n}^{0}\right) \cdot d_{n} \in \mathscr{R}$ is defined above and $k$ is a finite positive integer. Further, its semilocal convergence analysis is established by using two different approaches namely recurrent relations and recurrent functions under the assumption that the first derivative satisfies a combination of the Lipschitz and the center-Lipschitz continuity conditions.

The aim of this paper is to develop the directional $\mathrm{k}$-step Newton methods ( $\mathrm{k}$ a positive integer) is developed for solving a single nonlinear equation in $n$ variables. Its semilocal convergence analysis is established by using two different approaches (recurrent relations [7] and recurrent functions [9]) under the assumption that the first derivative satisfies a combination of the Lipschitz and the center-Lipschitz continuity conditions instead of only Lipschitz condition. The convergence theorems for the existence and uniqueness of the solution for each of them are established. Numerical examples including nonlinear Hammerstein type integral equations are worked out and significantly improved results are obtained. It is shown that the second approach based on recurrent functions solves problems failed to be solved by first one using recurrent relations. This demonstrates the efficacy and applicability of these approaches. This work extends the directional one and two-step Newton methods for solving a single nonlinear equation in $n$ variables. Their semilocal convergence analysis using majorizing sequences are studied in [1] and [5] under the assumption that the first derivative satisfies the Lipschitz and the combination of the Lipschitz and the center-Lipschitz continuity conditions, respectively. Finally, the computational order of convergence and the computational efficiency of developed method are studied. 
The paper is arranged as follows. Introduction is given in Section 1. The semilocal convergence analysis of (4) using recurrent relations is established in Section 2. In Section 3, the semilocal convergence analysis of (4) is established using recurrent functions. Some special cases and applications of (4) are also presented. In Section 4, numerical examples including nonlinear Hammerstein type integral equations are solved to demonstrate the applicability of our approaches. In Section 5, computational order of convergence and computational efficiency for (4) are described. Finally conclusions are given in Section 6.

\section{Recurrent relations for semilocal convergence analysis}

In this section, we shall use recurrent relations for establishing the semilocal convergence of (4) under the assumption that the first derivative satisfies a combination of the Lipschitz and the center-Lipschitz continuity conditions. Let $\beta, \eta, M_{0}$ and $M$ are positive constants. Assume that the following conditions hold.

$$
\begin{array}{ll}
\mathscr{B}_{1} & \left|\mathscr{F}\left(x_{0}^{0}\right)\right| \leq\left|\nabla \mathscr{F}\left(x_{0}^{0}\right) \cdot d_{0}\right| \eta \\
\mathscr{B}_{2} & \|\nabla \mathscr{F}(x)-\nabla \mathscr{F}(y)\| \leq M\|x-y\| \\
\mathscr{B}_{3} & \left\|\nabla \mathscr{F}(x)-\nabla \mathscr{F}\left(x_{0}^{0}\right)\right\| \leq M_{0}\left\|x-x_{0}^{0}\right\| \\
\mathscr{B}_{4} & \left\|\nabla \mathscr{F}\left(x_{0}^{0}\right)\right\| \geq \frac{1}{\beta} \\
\mathscr{B}_{5} & \angle\left(d_{n}, \nabla \mathscr{F}\left(x_{n}^{0}\right)\right) \leq \angle\left(d_{0}, \nabla \mathscr{F}\left(x_{0}^{0}\right)\right) \\
\mathscr{B}_{6} & L=M\left|\nabla \mathscr{F}\left(x_{0}^{0}\right) \cdot d_{0}\right|^{-1} \text { and } L_{0}=M_{0}\left\|\nabla \mathscr{F}\left(x_{0}^{0}\right)\right\|^{-1}
\end{array}
$$

Here, $\angle$ denotes the angle between two vectors $p$ and $q$, given by $\angle(p, q)=\arccos \frac{p \cdot q}{\|p\|\|q\|}, p \neq 0, q \neq 0$. $\mathscr{B}_{5}$ is equivalent to the condition

$$
\frac{\left|\nabla \mathscr{F}\left(x_{n}^{0}\right) \cdot d_{n}\right|}{\left\|\nabla \mathscr{F}\left(x_{n}^{0}\right)\right\|} \geq \frac{\left|\nabla \mathscr{F}\left(x_{0}^{0}\right) \cdot d_{0}\right|}{\left\|\nabla \mathscr{F}\left(x_{0}^{0}\right)\right\|}
$$

We shall call conditions $\left(\mathscr{B}_{1}-\mathscr{B}_{6}\right)$ as $\mathscr{B}$-condition throughout this study. Denote $\mathscr{B}\left(x^{*}, R\right)$ and $\overline{\mathscr{B}}\left(x^{*}, R\right)$ as open and closed balls with center at $x^{*}$ and radius $R$, respectively.

Lemma 1. Define $\tilde{b}_{R}=\frac{1}{1-M_{0} \beta R \eta}, a_{0}=L \tilde{b}_{R} \eta, s_{0}=a_{0} R$, where $R>1$ be a smallest positive real number satisfying $R\left(1-\frac{s_{0}^{k-1} a_{0}}{2}\right)=1+\frac{a_{0}}{2} \frac{1-s_{0}^{k-1}}{1-s_{0}}$, where $k$ be a finite positive integer. If $M_{0} \beta R \eta<1$ and $\frac{s_{0}^{k-1} a_{0}}{2}<1$, then starting with

$$
\begin{aligned}
t_{0,0}= & 0 \\
t_{0,1}= & \eta \\
t_{0,2}= & t_{0,1}+\frac{L \tilde{b}_{R}}{2}\left(t_{0,1}-t_{0,0}\right)^{2} \\
t_{0,3}= & t_{0,2}+\frac{L \tilde{b}_{R}}{2}\left(\left(t_{0,2}-t_{0,0}\right)+\left(t_{0,1}-t_{0,0}\right)\right)\left(t_{0,2}-t_{0,1}\right) \\
& \cdots \quad \cdots \ldots \ldots \\
t_{0, k}= & t_{0, k-1}+\frac{L \tilde{b}_{R}}{2}\left(\left(t_{0, k-1}-t_{0,0}\right)+\left(t_{0, k-2}-t_{0,0}\right)\right)\left(t_{0, k-1}-t_{0, k-2}\right)
\end{aligned}
$$


the sequence $\left\{t_{n, i}\right\}$, for $n \geq 1$ and $i=1,2, \ldots, k$ given by

$$
\begin{aligned}
t_{n, i} & =t_{n, i-1}+\frac{L \tilde{b}_{R}}{2}\left(\left(t_{n, i-1}-t_{n, 0}\right)+\left(t_{n, i-2}-t_{n, 0}\right)\right)\left(t_{n, i-1}-t_{n, i-2}\right) \text { for } i \neq 2 \text { and } \\
t_{n, i} & =t_{n, i-1}+\frac{L \tilde{b}_{R}}{2}\left(t_{n, i-1}-t_{n, i-2}\right)^{2} \text { for } i=2
\end{aligned}
$$

with $t_{n+1,0}=t_{n, k}$, is well defined, increasing, belongs to $(0, R \eta)$ and converges to its least upper bound $t^{*} \in(0, R \eta)$.

Proof. We shall use the mathematical induction to prove this lemma. From the assumptions of the lemma, we get $R>1$ and $t_{0,1}-t_{0,0}=\eta<R \eta$. Now,

$$
\begin{aligned}
t_{0,2}-t_{0,1} & =\frac{L \tilde{b}_{R}}{2}\left(t_{0,1}-t_{0,0}\right)^{2} \\
& =\frac{L \tilde{b}_{R} \eta}{2}\left(t_{0,1}-t_{0,0}\right)=\frac{a_{0}}{2}\left(t_{0,1}-t_{0,0}\right) .
\end{aligned}
$$

This gives

$$
t_{0,2}-t_{0,0}=t_{0,2}-t_{0,1}+t_{0,1}-t_{0,0}=\left(1+\frac{a_{0}}{2}\right) \eta<R \eta
$$

Now, from (6), (7), we get

$$
\begin{aligned}
t_{0,3}-t_{0,2} & =\frac{L \tilde{b}_{R}}{2}\left(\left(t_{0,2}-t_{0,0}\right)+\left(t_{0,1}-t_{0,0}\right)\right)\left(t_{0,2}-t_{0,1}\right) \\
& =\frac{L \tilde{b}_{R}}{2}(R \eta+R \eta)\left(t_{0,2}-t_{0,1}\right) \\
& =a_{0} R\left(t_{0,2}-t_{0,1}\right)=s_{0}\left(t_{0,2}-t_{0,1}\right)
\end{aligned}
$$

and

$$
\begin{aligned}
t_{0,3}-t_{0,0} & =t_{0,3}-t_{0,2}+t_{0,2}-t_{0,1}+t_{0,1}-t_{0,0} \\
& =s_{0}\left(t_{0,2}-t_{0,1}\right)+\frac{a_{0}}{2}\left(t_{0,1}-t_{0,0}\right)+\left(t_{0,1}-t_{0,0}\right) \\
& =s_{0} \frac{a_{0}}{2}\left(t_{0,1}-t_{0,0}\right)+\frac{a_{0}}{2}\left(t_{0,1}-t_{0,0}\right)+\left(t_{0,1}-t_{0,0}\right) \\
& =\left(\left(1+s_{0}\right) \frac{a_{0}}{2}+1\right)\left(t_{0,1}-t_{0,0}\right)<R \eta
\end{aligned}
$$

Now, for $3 \leq i \leq k-2$, we get

$$
\begin{aligned}
t_{0, i+1}-t_{0, i} & =\frac{L \tilde{b}_{R}}{2}\left(\left(t_{0, i}-t_{0,0}\right)+\left(t_{0, i-1}-t_{0,0}\right)\right)\left(t_{0, i}-t_{0, i-1}\right) \\
& \leq \frac{L \tilde{b}_{R}}{2}(R \eta+R \eta)\left(t_{0, i}-t_{0, i-1}\right) \\
& =a_{0} R\left(t_{0, i}-t_{0, i-1}\right)=s_{0}\left(t_{0, i}-t_{0, i-1}\right)
\end{aligned}
$$

This gives

$$
t_{0, i+1}-t_{0, i} \leq s_{0}\left(t_{0, i}-t_{0, i-1}\right) \leq s_{0}^{i-1}\left(t_{0,2}-t_{0,1}\right)=\frac{a_{0}}{2} s_{0}^{i-1}\left(t_{0,1}-t_{0,0}\right) .
$$

Using (8), (9) and (10), for $i=k-1$, we get $t_{0, k}-t_{0, k-1} \leq \frac{a_{0}}{2} s_{0}^{k-2}\left(t_{0,1}-t_{0,0}\right)$. Thus,

$$
t_{0, k}-t_{0,0}=t_{0, k}-t_{0, k-1}+t_{0, k-1}-t_{0, k-2}+\ldots+t_{0,1}-t_{0,0} \leq\left(1+\frac{a_{0}}{2}\left(1+\ldots+s_{0}^{k-2}\right)\right) \eta<R \eta
$$


This shows that $t_{0, k} \in(0, R \eta)$ for all $\mathrm{k}$ and thus lemma holds true for $n=0$. In order to show that lemma holds for $n \geq 1$, we take $\eta_{0}=\eta$ and construct some scalar sequences defined for $n \geq 1$ by $\eta_{n}=\frac{s_{n-1}^{k-1} a_{n-1} \eta_{n-1}}{2}, a_{n}=L \tilde{b}_{R} \eta_{n}$ and $s_{n}=a_{n} R$. For $n=1$, using (10), (11), we get

$$
\begin{aligned}
t_{1,1}-t_{1,0} & =\frac{L \tilde{b}_{R}}{2}\left(\left(t_{0, k}-t_{0,0}\right)+\left(t_{0, k-1}-t_{0,0}\right)\right)\left(t_{0, k}-t_{0, k-1}\right) \\
& \leq \frac{L \tilde{b}_{R}}{2}(R \eta+R \eta)\left(t_{0, k}-t_{0, k-1}\right) \\
& \leq a_{0} R s_{0}^{k-2}\left(t_{0,2}-t_{0,1}\right)=\frac{s_{0}^{k-1} a_{0} \eta_{0}}{2}=\eta_{1}<R \eta_{1}
\end{aligned}
$$

and

$$
\begin{aligned}
t_{1,1}-t_{0,0} & =\left(t_{1,1}-t_{1,0}\right)+\left(t_{1,0}-t_{0,0}\right) \\
& \leq s_{0}^{k-1} \frac{a_{0}}{2} \eta+\left(1+\frac{a_{0}}{2}\left(1+\ldots+s_{0}^{k-2}\right)\right) \eta \\
& =\left(1+\frac{a_{0}}{2} \sum_{r=1}^{r=k} s_{0}^{r-1}\right) \eta<R \eta
\end{aligned}
$$

This gives

$$
\begin{aligned}
t_{1,2}-t_{1,1} & =\frac{L \tilde{b}_{R}}{2}\left(t_{1,1}-t_{1,0}\right)^{2} \\
& =\frac{L \tilde{b}_{R}}{2} \eta_{1}\left(t_{1,1}-t_{1,0}\right)=\frac{a_{1}}{2}\left(t_{1,1}-t_{1,0}\right)
\end{aligned}
$$

Thus, from (12) and (13), we get

$$
t_{1,2}-t_{1,0}=t_{1,2}-t_{1,1}+t_{1,1}-t_{1,0} \leq\left(1+\frac{a_{1}}{2}\right)\left(t_{1,1}-t_{1,0}\right)=\left(1+\frac{a_{1}}{2}\right) \eta_{1}
$$

and

$$
\begin{aligned}
t_{1,2}-t_{0,0} & =t_{1,2}-t_{1,0}+t_{1,0}-t_{0,0} \\
& \leq\left(1+\frac{a_{1}}{2}\right) \eta_{1}+\left(1+\frac{a_{0}}{2} \sum_{r=1}^{r=k-1} s_{0}^{r-1}\right) \eta
\end{aligned}
$$

This gives

$$
\begin{aligned}
t_{1,3}-t_{1,2} & =\frac{L \tilde{b}_{R}}{2}\left(\left(t_{1,2}-t_{1,0}\right)+\left(t_{1,1}-t_{1,0}\right)\right)\left(t_{1,2}-t_{1,1}\right) \\
& \leq L \tilde{b}_{R} R \eta_{1}\left(t_{1,2}-t_{1,1}\right)=a_{1} R\left(t_{1,2}-t_{1,1}\right)=s_{1}\left(t_{1,2}-t_{1,1}\right)
\end{aligned}
$$

Now, proceeding in a similar manner, we get

$$
t_{1, i+1}-t_{1, i} \leq s_{1}^{i-1}\left(t_{1,2}-t_{1,1}\right), \quad \text { for } \quad 3 \leq i \leq k-1 .
$$

This gives

$$
t_{1, k}-t_{1,0} \leq\left(1+\frac{a_{1}}{2}\left(1+\ldots+s_{1}^{k-2}\right)\right) \eta_{1}
$$


Using the assumptions of the lemma, it is easy to see that $\eta_{1}<\eta, a_{1}<a_{0}$ and $s_{1}<s_{0}$. Now, using (11) and (14), we get

$$
\begin{aligned}
t_{1, k}-t_{0,0} & =t_{1, k}-t_{1,0}+t_{1,0}-t_{0,0} \\
& \leq\left(1+\frac{a_{1}}{2}\left(1+\ldots+s_{1}^{k-2}\right)\right) \eta_{1}+\left(1+\frac{a_{0}}{2}\left(1+\ldots+s_{0}^{k-2}\right)\right) \eta \\
& <\left(\eta_{1}+\eta\right)\left(1+\frac{a_{0}}{2}\left(1+\ldots+s_{0}^{k-2}\right)\right) \\
& =\left(\frac{s_{0}^{k-1} a_{0}}{2}+1\right)\left(1+\frac{a_{0}}{2}\left(1+\ldots+s_{0}^{k-2}\right)\right) \eta<R \eta
\end{aligned}
$$

Thus, $t_{1, k} \in(0, R \eta)$ and hence lemma holds for $n=1$. Now, proceeding in a similar manner, it can be easily established that

$$
\begin{aligned}
t_{n, k}-t_{0,0} & \leq\left(1+\frac{a_{n}}{2}\left(1+s_{n}+\ldots+s_{n}^{k-2}\right)\right) \eta_{n}+\left(1+\frac{a_{n-1}}{2}\left(1+s_{n-1}+\ldots+s_{n-1}^{k-2}\right)\right) \eta_{n-1} \\
& +\ldots+\left(1+\frac{a_{0}}{2}\left(1+s_{0}+\ldots+s_{0}^{k-2}\right)\right) \eta \\
& =\sum_{j=0}^{n}\left(1+\frac{a_{j}}{2}\left(\sum_{r=1}^{k-1} s_{j}^{r-1}\right)\right) \eta_{j} .
\end{aligned}
$$

Now, in order to show $t_{n, i} \in(0, R \eta)$, we have to show that the sequences defined by $a_{j}, s_{j}$ and $\eta_{j}$ for $j>1$ are decreasing. From the assumption of the lemma, we have $\eta_{2}=\frac{s_{1}^{k-1} a_{1} \eta_{1}}{2}<\eta_{1}, a_{2}=L \tilde{b}_{R} \eta_{2}<$ $L \tilde{b}_{R} \eta_{1}=a_{1}$ and $s_{2}=a_{2} R<a_{1} R=s_{1}$. Proceeding in a similar way and using mathematical induction on $j$, it can be easily proved that $a_{j}, s_{j}$ and $\eta_{j}$ are decreasing for any $j$. Now, using (15) and properties of sequences $a_{j}, s_{j}$ and $\eta_{j}$, we get

$$
\begin{aligned}
t_{n, k}-t_{0,0} & \leq \sum_{j=0}^{n}\left(1+\frac{a_{j}}{2}\left(\sum_{r=1}^{k-1} s_{j}^{r-1}\right)\right) \eta_{j} \\
& \leq\left[1+\frac{a_{0}}{2}\left(1+s_{0}+\ldots+s_{0}^{k-2}\right)\right] \sum_{j=0}^{n} \eta_{j} \\
& \leq\left[1+\frac{a_{0}}{2}\left(1+s_{0}+\ldots+s_{0}^{k-2}\right)\right] \sum_{j=0}^{n}\left(s_{0}^{k-1} \frac{a_{0}}{2}\right)^{j} \eta .
\end{aligned}
$$

Using (16), we get

$$
t_{n, k}-t_{0,0} \rightarrow \frac{\left(1+\frac{a_{0}}{2}\left(\frac{1-s_{0}^{k-1}}{1-s_{0}}\right)\right)}{1-\frac{s_{0}^{k-1} a_{0}}{2}} \eta=R \eta \text { as } n \rightarrow \infty
$$

This shows that $t_{n, k} \in(0, R \eta)$ for all $n$ and $k$. It remains to show that $\left\{t_{n, k}\right\}$ is a convergent sequence. For 
this, it is sufficient to show that $\left\{t_{n, k}\right\}$ is a Cauchy sequence. For this, using previous results, we get

$$
\begin{aligned}
t_{n+1, k}-t_{n, k} & =t_{n+1, k}-t_{n+1, k-1}+\ldots+t_{n+1,1}-t_{n+1,0} \quad\left(\text { since } t_{n+1,0}=t_{n, k} \forall n \geq 0\right) \\
& \leq s_{n+1}^{k-2}\left(t_{n+1,2}-t_{n+1,1}\right)+s_{n+1}^{k-3}\left(t_{n+1,2}-t_{n+1,1}\right)+\ldots+\left(t_{n+1,2}-t_{n+1,1}\right)+t_{n+1,1}-t_{n+1,0} \\
& =\left(1+s_{n+1}+\ldots+s_{n+1}^{k-2}\right)\left(t_{n+1,2}-t_{n+1,1}\right)+t_{n+1,1}-t_{n+1,0} \\
& \leq\left(\left(1+s_{n+1}+\ldots+s_{n+1}^{k-2}\right) \frac{a_{n+1}}{2}+1\right)\left(t_{n+1,1}-t_{n+1,0}\right) \\
& \leq\left(\frac{1-s_{0}^{k-1}}{1-s_{0}} \frac{a_{0}}{2}+1\right) \eta_{n+1} .
\end{aligned}
$$

Thus, for a fixed $m \geq 1$,

$$
\begin{aligned}
t_{n+m, k}-t_{m, k} & =\sum_{i=1}^{i=n}\left(t_{m+i, k}-t_{m+i-1, k}\right) \\
& \leq\left(\frac{1-s_{0}^{k-1}}{1-s_{0}} \frac{a_{0}}{2}+1\right) \sum_{i=1}^{i=n} \eta_{m+i} \\
& \leq\left(\frac{1-s_{0}^{k-1}}{1-s_{0}} \frac{a_{0}}{2}+1\right) \sum_{i=1}^{i=n}\left(\frac{s_{0}^{k-1} a_{0}}{2}\right)^{m+i} \rightarrow 0, \text { as } n \rightarrow \infty .
\end{aligned}
$$

This shows that $\left\{t_{n, k}\right\}$ is increasing, bounded and converges to some $t^{*} \in(0, R \eta)$.

Theorem 1. Let $\mathscr{F}: \mathscr{D} \subset \mathscr{R}^{n} \rightarrow \mathscr{R}$ be a Fréchet-differentiable mapping satisfying $\mathscr{B}$-condition. Assume that the conditions of Lemma 1 hold with $\left\|d_{n}\right\|=1$. Starting with $x_{0}^{0} \in \mathscr{D}$, the sequence $\left\{x_{n}^{i}\right\}, n \geq 0, i=$ $1, \ldots, k$ generated by (4) is well defined, belongs to $\overline{\mathscr{B}}\left(x_{0}^{0}, R \eta\right)$ and converges to $x^{*} \in \overline{\mathscr{B}}\left(x_{0}^{0}, R \eta\right)$.

Proof. For any $x \in \mathscr{D} \subset \mathscr{B}\left(x_{0}^{0}, R \eta\right)$, we have

$$
\nabla \mathscr{F}\left(x_{0}^{0}\right)=\nabla \mathscr{F}\left(x_{0}^{0}\right)-\nabla \mathscr{F}(x)+\nabla \mathscr{F}(x)
$$

Taking norm on both sides, we get

$$
\left\|\nabla \mathscr{F}\left(x_{0}^{0}\right)\right\| \leq\left\|\nabla \mathscr{F}\left(x_{0}^{0}\right)-\nabla \mathscr{F}(x)\right\|+\|\nabla \mathscr{F}(x)\|
$$

This gives

$$
\begin{aligned}
\|\nabla \mathscr{F}(x)\| & \geq\left\|\nabla \mathscr{F}\left(x_{0}^{0}\right)\right\|-\left\|\nabla \mathscr{F}\left(x_{0}^{0}\right)-\nabla \mathscr{F}(x)\right\| \\
& \geq\left\|\nabla \mathscr{F}\left(x_{0}^{0}\right)\right\|\left(1-M_{0}\left\|\nabla \mathscr{F}\left(x_{0}^{0}\right)\right\|^{-1}\left\|x-x_{0}^{0}\right\|\right) \\
& \geq\left\|\nabla \mathscr{F}\left(x_{0}^{0}\right)\right\|\left(1-M_{0} \beta R \eta\right)
\end{aligned}
$$

Thus, we have

$$
\|\nabla \mathscr{F}(x)\|^{-1} \leq \frac{\left\|\nabla \mathscr{F}\left(x_{0}^{0}\right)\right\|^{-1}}{1-M_{0} \beta R \eta}=\left\|\nabla \mathscr{F}\left(x_{0}^{0}\right)\right\|^{-1} \tilde{b}_{R} .
$$

From (5) and (17), we get

$$
\frac{1}{|\nabla \mathscr{F}(x) \cdot d|} \leq \frac{\left\|\nabla \mathscr{F}\left(x_{0}^{0}\right)\right\|}{\|\nabla \mathscr{F}(x)\|\left|\nabla \mathscr{F}\left(x_{0}^{0}\right) \cdot d_{0}\right|} \leq \frac{\tilde{b}_{R}}{\left|\nabla \mathscr{F}\left(x_{0}^{0}\right) \cdot d_{0}\right|} .
$$


Also, from (4) for $i=1, \ldots, k$ and $n \geq 0$, we get

$$
x_{n}^{i}=x_{n}^{i-1}+h_{n}^{i-1}, \text { where } h_{n}^{i-1}=-\frac{\mathscr{F}\left(x_{n}^{i-1}\right)}{\nabla \mathscr{F}\left(x_{n}^{0}\right) \cdot d_{n}} d_{n}
$$

Using Lemma 1, $\mathscr{B}$-condition, (4) and (19), we get $\left\|x_{0}^{1}-x_{0}^{0}\right\| \leq \eta=t_{0,1}-t_{0,0} \leq R \eta$. Also,

$$
\begin{aligned}
\mathscr{F}\left(x_{0}^{1}\right) & =\mathscr{F}\left(x_{0}^{1}\right)-\mathscr{F}\left(x_{0}^{0}\right)+\mathscr{F}\left(x_{0}^{0}\right) \\
& =\mathscr{F}\left(x_{0}^{1}\right)-\mathscr{F}\left(x_{0}^{0}\right)+\frac{\mathscr{F}\left(x_{0}^{0}\right)}{\nabla \mathscr{F}\left(x_{0}^{0}\right) \cdot d_{0}} d_{0} \cdot \nabla \mathscr{F}\left(x_{0}^{0}\right) \\
& =\mathscr{F}\left(x_{0}^{1}\right)-\mathscr{F}\left(x_{0}^{0}\right)-h_{0}^{0} \nabla \mathscr{F}\left(x_{0}^{0}\right) \\
& =\int_{0}^{1}\left(\nabla \mathscr{F}\left(x_{0}^{0}+\theta\left(x_{0}^{1}-x_{0}^{0}\right)\right)-\nabla \mathscr{F}\left(x_{0}^{0}\right)\right) d \theta\left(x_{0}^{1}-x_{0}^{0}\right)
\end{aligned}
$$

On taking norm on both sides, we get

$$
\left|\mathscr{F}\left(x_{0}^{1}\right)\right| \leq \frac{M_{0}}{2}\left\|x_{0}^{1}-x_{0}^{0}\right\|^{2}
$$

Using $\mathscr{B}_{6},(18),(19),(20)$ and (21), we get

$$
\begin{aligned}
\left\|x_{0}^{2}-x_{0}^{1}\right\| & =\left\|h_{0}^{1}\right\|=\frac{\left|\mathscr{F}\left(x_{0}^{1}\right)\right|}{\left|\nabla \mathscr{F}\left(x_{0}^{0}\right) \cdot d_{0}\right|} \\
& \leq \frac{L}{2}\left\|x_{0}^{1}-x_{0}^{0}\right\|^{2}<\frac{L}{2} \tilde{b}_{R}\left(t_{0,1}-t_{0,0}\right)^{2}=t_{0,2}-t_{0,1}
\end{aligned}
$$

and

$$
\left\|x_{0}^{2}-x_{0}^{0}\right\| \leq\left\|x_{0}^{2}-x_{0}^{1}\right\|+\left\|x_{0}^{1}-x_{0}^{0}\right\| \leq t_{0,2}-t_{0,1}+t_{0,1}-t_{0,0}=t_{0,2}
$$

Using Lemma 1 , we get $x_{0}^{2} \in \overline{\mathscr{B}}\left(x_{0}^{0}, R \eta\right)$. Now, using (4) and (22), we get

$$
\begin{aligned}
\mathscr{F}\left(x_{0}^{2}\right) & =\mathscr{F}\left(x_{0}^{1}\right)+\nabla \mathscr{F}\left(x_{0}^{1}\right)\left(x_{0}^{2}-x_{0}^{1}\right)+\int_{0}^{1}\left(\nabla \mathscr{F}\left(x_{0}^{1}+\theta\left(x_{0}^{2}-x_{0}^{1}\right)\right)-\nabla \mathscr{F}\left(x_{0}^{1}\right)\right)\left(x_{0}^{2}-x_{0}^{1}\right) d \theta \\
= & \left(\nabla \mathscr{F}\left(x_{0}^{1}\right)-\nabla \mathscr{F}\left(x_{0}^{0}\right)\right)\left(x_{0}^{2}-x_{0}^{1}\right)+\int_{0}^{1}\left(\nabla \mathscr{F}\left(x_{0}^{1}+\theta\left(x_{0}^{2}-x_{0}^{1}\right)\right)-\nabla \mathscr{F}\left(x_{0}^{1}\right)\right)\left(x_{0}^{2}-x_{0}^{1}\right) d \theta
\end{aligned}
$$

Taking norm on both sides, we get

$$
\left|\mathscr{F}\left(x_{0}^{2}\right)\right| \leq M_{0}\left\|x_{0}^{1}-x_{0}^{0}\right\|\left\|x_{0}^{2}-x_{0}^{1}\right\|+\frac{M}{2}\left\|x_{0}^{2}-x_{0}^{1}\right\|^{2} .
$$

Using (4), (18), (23) and (24), we get

$$
\begin{aligned}
\left\|x_{0}^{3}-x_{0}^{2}\right\| & =\left\|h_{0}^{2}\right\| \leq \frac{\left|\mathscr{F}\left(x_{0}^{2}\right)\right|}{\left|\nabla \mathscr{F}\left(x_{0}^{0}\right) \cdot d_{0}\right|} \\
& \leq \frac{M}{\left|\nabla \mathscr{F}\left(x_{0}^{0}\right) \cdot d_{0}\right|}\left(\left(t_{0,1}-t_{0,0}\right)\left(t_{0,2}-t_{0,1}\right)+\frac{1}{2}\left(t_{0,2}-t_{0,1}\right)^{2}\right) \\
& =\frac{M}{2\left|\nabla \mathscr{F}\left(x_{0}^{0}\right) \cdot d_{0}\right|}\left[2\left(t_{0,1}-t_{0,0}\right)+\left(t_{0,2}-t_{0,1}\right)\right]\left(t_{0,2}-t_{0,1}\right) \\
& <\frac{L \tilde{b}_{R}}{2}\left[\left(t_{0,1}-t_{0,0}\right)+\left(t_{0,2}-t_{0,0}\right)\right]\left(t_{0,2}-t_{0,1}\right)=t_{0,3}-t_{0,2} .
\end{aligned}
$$


Combining (21) to (25), this gives,

$$
\begin{aligned}
\left\|x_{0}^{3}-x_{0}^{0}\right\| & \leq\left\|x_{0}^{3}-x_{0}^{2}\right\|+\left\|x_{0}^{2}-x_{0}^{1}\right\|+\left\|x_{0}^{1}-x_{0}^{0}\right\| \\
& \leq t_{0,3}-t_{0,2}+t_{0,2}-t_{0,1}+t_{0,1}-t_{0,0}=t_{0,3}-t_{0,0}
\end{aligned}
$$

and proceeding in a similar way for $3 \leq i \leq k$, we get

$$
\left\|x_{0}^{i}-x_{0}^{i-1}\right\| \leq t_{0, i}-t_{0, i-1} \text { and }\left\|x_{0}^{i}-x_{0}^{0}\right\| \leq t_{0, i}-t_{0,0} .
$$

As $x_{1}^{0}=x_{0}^{k}$,

$$
\begin{aligned}
\mathscr{F}\left(x_{1}^{0}\right) & =\mathscr{F}\left(x_{0}^{k-1}\right)+\nabla \mathscr{F}\left(x_{0}^{k-1}\right)\left(x_{0}^{k}-x_{0}^{k-1}\right)+\int_{0}^{1}\left(\nabla \mathscr{F}\left(x_{0}^{k-1}+\theta\left(x_{0}^{k}-x_{0}^{k-1}\right)\right)-\nabla F\left(x_{0}^{k-1}\right)\right) d \theta\left(x_{0}^{k}-x_{0}^{k-1}\right) \\
& =\left(\nabla \mathscr{F}\left(x_{0}^{k-1}\right)-\nabla \mathscr{F}\left(x_{0}^{0}\right)\right)\left(x_{0}^{k}-x_{0}^{k-1}\right)+\int_{0}^{1}\left(\nabla \mathscr{F}\left(x_{0}^{k-1}+\theta\left(x_{0}^{k}-x_{0}^{k-1}\right)\right)-\nabla F\left(x_{0}^{k-1}\right)\right) d \theta\left(x_{0}^{k}-x_{0}^{k-1}\right)
\end{aligned}
$$

Taking norm on both sides, this gives

$$
\begin{aligned}
\left|\mathscr{F}\left(x_{1}^{0}\right)\right| & \leq M_{0}\left\|x_{0}^{k-1}-x_{0}^{0}\right\|\left\|x_{0}^{k}-x_{0}^{k-1}\right\|+\frac{M}{2}\left\|x_{0}^{k}-x_{0}^{k-1}\right\|^{2} \\
& \leq\left(M\left\|x_{0}^{k-1}-x_{0}^{0}\right\|+\frac{M}{2}\left\|x_{0}^{k}-x_{0}^{k-1}\right\|\right)\left\|x_{0}^{k}-x_{0}^{k-1}\right\| \\
& \leq M\left(\left(t_{0, k-1}-t_{0,0}\right)+\frac{1}{2}\left(t_{0, k}-t_{0, k-1}\right)\right)\left(t_{0, k}-t_{0, k-1}\right) .
\end{aligned}
$$

Using $\mathscr{B}_{6}$, (4), (18) and (26), we get

$$
\begin{aligned}
\left\|x_{1}^{1}-x_{1}^{0}\right\| & =\left\|h_{1}^{0}\right\| \leq \frac{M}{\left|\nabla \mathscr{F}\left(x_{0}^{0}\right) \cdot d_{0}\right|}\left(\left(t_{0, k-1}-t_{0,0}\right)+\frac{1}{2}\left(t_{0, k}-t_{0, k-1}\right)\right)\left(t_{0, k}-t_{0, k-1}\right) \\
& =\frac{L \tilde{b}_{R}}{2}\left(2\left(t_{0, k-1}-t_{0,0}\right)+\left(t_{0, k}-t_{0, k-1}\right)\right)\left(t_{0, k}-t_{0, k-1}\right)=t_{1,1}-t_{0, k}=t_{1,1}-t_{1,0} .
\end{aligned}
$$

Thus, $\left\|x_{1}^{1}-x_{0}^{0}\right\| \leq t_{1,1}-t_{0,0}$. So, from Lemma $1, x_{1}^{1} \in \overline{\mathscr{B}}\left(x_{0}^{0}, R \eta\right)$ and repeating the same procedure as in (20), we get

$$
\left|\mathscr{F}\left(x_{1}^{1}\right)\right| \leq \frac{M}{2}\left\|x_{1}^{1}-x_{1}^{0}\right\|^{2} .
$$

Now, using (4), $\mathscr{B}$-condition, (17) and (27), we get

$$
\begin{aligned}
\left\|x_{1}^{2}-x_{1}^{1}\right\| & \leq \frac{M \tilde{b}_{R}}{2}\left\|x_{1}^{1}-x_{1}^{0}\right\|^{2}\left|\nabla \mathscr{F}\left(x_{0}^{0}\right) \cdot d_{0}\right|^{-1} \\
& \leq \frac{L \tilde{b}_{R}}{2}\left(t_{1,1}-t_{1,0}\right)^{2}=t_{1,2}-t_{1,1}
\end{aligned}
$$

Proceeding in the similar way, we get

$$
\left\|\mathscr{F}\left(x_{n}^{0}\right)\right\| \leq M\left\|x_{n-1}^{k-1}-x_{n-1}^{0}\right\|\left\|x_{n-1}^{k}-x_{n-1}^{k-1}\right\|+\frac{M}{2}\left\|x_{n-1}^{k}-x_{n-1}^{k-1}\right\|^{2} .
$$

and from (4), $\mathscr{B}_{6}$ and (19), we get

$$
\begin{aligned}
\left\|x_{n}^{1}-x_{n}^{0}\right\| & =\left\|h_{n}^{0}\right\| \leq \frac{\left|\mathscr{F}\left(x_{n}^{0}\right)\right|}{\left|\nabla \mathscr{F}\left(x_{n}^{0}\right) \cdot d_{n}\right|} \\
& \leq M \tilde{b}_{R}\left|\nabla \mathscr{F}\left(x_{0}\right) \cdot d_{0}\right|^{-1}\left(\left(t_{n-1, k-1}-t_{n-1,0}\right)\left(t_{n-1, k}-t_{n-1, k-1}\right)+\frac{1}{2}\left(t_{n-1, k}-t_{n-1, k-1}\right)^{2}\right) \\
& =\frac{L \tilde{b}_{R}}{2}\left(\left(t_{n-1, k-1}-t_{n-1,0}\right)+\left(t_{n-1, k}-t_{n-1,0}\right)\right)\left(t_{n-1, k}-t_{n-1, k-1}\right)=t_{n, 1}-t_{n, 0} .
\end{aligned}
$$


In the similar manner, we can get

$$
\left\|x_{n}^{2}-x_{n}^{1}\right\| \leq \frac{L \tilde{b}_{R}}{2}\left\|x_{n}^{1}-x_{n}^{0}\right\|^{2} \leq \frac{L \tilde{b}_{R}}{2}\left(t_{n, 1}-t_{n, 0}\right)^{2}=t_{n, 2}-t_{n, 1}
$$

Now, using mathematical induction on $n$, we can easily get

$$
\left\|x_{n}^{k}-x_{n}^{k-1}\right\| \leq \frac{L \tilde{b}_{R}}{2}\left[\left(t_{n, k-2}-t_{n, 0}\right)+\left(t_{n, k-1}-t_{n, 0}\right)\right]\left(t_{n, k-1}-t_{n, k-2}\right)=t_{n, k}-t_{n, k-1} .
$$

and

$$
\left\|\mathscr{F}\left(x_{n}^{k}\right)\right\| \leq \frac{M}{2}\left[\left(t_{n, k}-t_{n, 0}\right)+\left(t_{n, k-1}-t_{n, 0}\right)\right]\left(t_{n, k}-t_{n, k-1}\right) \rightarrow 0 \text { as } n \rightarrow \infty .
$$

Thus, using Lemma 1 and convergence of the sequence $\left\{t_{n, i}\right\}, n \geq 0, i=1, \ldots, k$, it follows that $\left\{x_{n, k}\right\}$ is a Cauchy sequence and hence it converges to some $x^{*} \in \overline{\mathscr{B}}\left(x_{0}^{0}, R \eta\right)$. Since $\mathscr{F}$ is continuous it follows that $\mathscr{F}\left(x^{*}\right)=0$. Thus, $x^{*}$ is a solution of $(1)$.

\section{Recurrent functions for semilocal convergence analysis}

In this section, the semilocal convergence of (4) is established by recurrent functions under the assumption that the first derivative satisfies a combination of the Lipschitz and the center-Lipschitz continuity conditions. It is assumed here that the $\mathscr{B}$-condition as described in Section 2 holds.

Lemma 2. Let $L_{0}, L$ and $\eta$ are given positive constants. Taking $l_{0,0}=0$ and $l_{0,1}=\eta$, define a scalar sequence $\left\{l_{n, i}\right\}$, for $n \geq 0$ and $i=1, \ldots, k$ given by

$$
\begin{aligned}
l_{n, 2} & =l_{n, 1}+\frac{L}{2} \frac{\left(l_{n, 1}-l_{n, 0}\right)^{2}}{1-L_{0} l_{n, 0}}, \text { for } i=2, \\
l_{n, i} & =l_{n, i-1}+\frac{L}{2} \frac{\left[\left(l_{n, i-1}-l_{n, 0}\right)+\left(l_{n, i-2}-l_{n, 0}\right)\right]}{1-L_{0} l_{n, 0}}\left(l_{n, i-1}-l_{n, i-2}\right) \text { for } i \neq 2, \\
l_{n, k} & =l_{n+1,0} .
\end{aligned}
$$

Let $\alpha$ be the smallest positive root of the function $H_{k}(t)$ defined on $(0,1)$ by

$$
H_{k}(t)=L\left(t^{k}-1\right)\left(\frac{1-t^{k}}{1-t}+\frac{1-t^{k-1}}{1-t}\right)+2 t^{k+1} L_{0}\left(\frac{1-t^{k}}{1-t}\right) .
$$

such that

$$
0 \leq \frac{L\left(l_{0, k}+l_{0, k-1}\right)}{1-L_{0} l_{0, k}}<\delta<2\left(1-L_{0} \eta\right)
$$

for $\delta=2 \alpha$. The scalar sequence defined by (28) is increasing, bounded above by $l^{* *}=\frac{\eta}{1-\alpha}$ and converges to the least upper bound $l^{*} \in\left(0, l^{* *}\right)$. Also, for all $n \geq 0$ and $i=1,2, \ldots, k$, we have

$$
l_{n, i-1} \leq l_{n, i}, L_{0} l^{*}<1 \text { and } 0 \leq\left(l_{n, i}-l_{n, i-1}\right) \leq \frac{\delta}{2}\left(l_{n, i-1}-l_{n, i-2}\right) .
$$

and

$$
0 \leq l_{n, i}-l_{n, 0} \leq\left(\frac{1-\left(\frac{\delta}{2}\right)^{i}}{1-\frac{\delta}{2}}\right)\left(l_{n, 1}-l_{n, 0}\right)
$$


Proof. $H_{k}(t)$ is continuous on $(0,1)$ and from (29), we get $H_{k}(0)<0$ and $H_{k}(1)>0$. By intermediate value theorem, there exists at least one root of $H_{k}(t)$ on $(0,1)$. Let the smallest root be denoted by $\alpha$. Now, we shall prove (31) by using mathematical induction on $n$. Using (28), for $n=0$ and $i=2$, we get

$$
l_{0,2}=l_{0,1}+\frac{L}{2}\left(l_{0,1}-l_{0,0}\right)^{2}
$$

This shows that $l_{0,2}>l_{0,1}$. Suppose it is true for all $i<k-1$, that is, $l_{0, i}>l_{0, i-1}$ and

$$
l_{0, i+1}-l_{0, i}=\frac{L}{2}\left(\left(l_{0, i}-l_{0,0}\right)+\left(l_{0, i-1}-l_{0,0}\right)\right)\left(l_{0, i}-l_{0, i-1}\right) .
$$

This shows that $l_{0, i+1}>l_{0, i}$ and

$$
\frac{L}{2}\left(\left(l_{0, i}-l_{0,0}\right)+\left(l_{0, i-1}-l_{0,0}\right)\right)\left(l_{0, i}-l_{0, i-1}\right)<\frac{L}{2} \frac{\left(\left(l_{0, k}-l_{0,0}\right)+\left(l_{0, k-1}-l_{0,0}\right)\right)}{1-L_{0} l_{0, k}}\left(l_{0, i}-l_{0, i-1}\right)
$$

Using (30) and (33), we get $\left(l_{0, i+1}-l_{0, i}\right)<\frac{\delta}{2}\left(l_{0, i}-l_{0, i-1}\right) \forall i=1, \ldots, k$. Thus, (31) holds true for $n=0$. Also, (31) and (32) are true for $n=r \geq 1$, if

$$
\left.\begin{array}{l}
0 \leq\left(l_{r, 2}-l_{r, 1}\right) \leq \frac{\delta}{2}\left(l_{r, 1}-l_{r, 0}\right) \\
0 \leq\left(l_{r, 3}-l_{r, 2}\right) \leq \frac{\delta}{2}\left(l_{r, 2}-l_{r, 1}\right) \\
\ldots \ldots \ldots \\
0 \leq\left(l_{r, k}-l_{r, k-1}\right) \leq \frac{\delta}{2}\left(l_{r, k-1}-l_{r, k-2}\right) \\
0 \leq\left(l_{r+1,1}-l_{r, k}\right) \leq \frac{\delta}{2}\left(l_{r, k}-l_{r, k-1}\right) .
\end{array}\right\}
$$

(34) is true, if

$$
\left(l_{r+1,1}-l_{r+1,0}\right)=\left(l_{r+1,1}-l_{r, k}\right) \leq\left(\frac{\delta}{2}\right)^{k}\left(l_{r, 1}-l_{r, 0}\right) .
$$

If (34) and (35) hold, then

$$
\begin{aligned}
l_{r+1,1} & \leq l_{r, k}+\left(\frac{\delta}{2}\right)^{k}\left(l_{r, 1}-l_{r, 0}\right) \\
& \leq l_{r, k-1}+\left(\frac{\delta}{2}\right)^{k-1}\left(l_{r, 1}-l_{r, 0}\right)+\left(\frac{\delta}{2}\right)^{k}\left(l_{r, 1}-l_{r, 0}\right) \\
& \leq l_{r, 1}+\left[\frac{\delta}{2}+\left(\frac{\delta}{2}\right)^{2}+\ldots+\left(\frac{\delta}{2}\right)^{k}\right]\left(l_{r, 1}-l_{r, 0}\right) \\
& =l_{r, 1}+\frac{\frac{\delta}{2}\left(1-\left(\frac{\delta}{2}\right)^{k}\right)}{1-\left(\frac{\delta}{2}\right)^{2}}\left(l_{r, 1}-l_{r, 0}\right)=l_{r, 0}+\frac{1-\left(\frac{\delta}{2}\right)^{k+1}}{1-\frac{\delta}{2}}\left(l_{r, 1}-l_{r, 0}\right)
\end{aligned}
$$


Now, if (35) and (36) hold then using some algebraic manipulation on $r$, we get

$$
\begin{aligned}
& l_{r+1,1} \leq l_{r, 1}+\frac{\frac{\delta}{2}\left(1-\left(\frac{\delta}{2}\right)^{k}\right)}{1-\left(\frac{\delta}{2}\right)}\left(l_{r, 1}-l_{r, 0}\right) \\
& \leq l_{r-1,1}+\frac{\frac{\delta}{2}\left(1-\left(\frac{\delta}{2}\right)^{k}\right)}{1-\left(\frac{\delta}{2}\right)}\left(l_{r-1,1}-l_{r-1,0}\right)+\frac{\frac{\delta}{2}\left(1-\left(\frac{\delta}{2}\right)^{k}\right)}{1-\left(\frac{\delta}{2}\right)}\left(l_{r, 1}-l_{r, 0}\right) \\
& \leq l_{0,1}+\frac{\frac{\delta}{2}\left(1-\left(\frac{\delta}{2}\right)^{k}\right)}{1-\left(\frac{\delta}{2}\right)}\left(l_{0,1}-l_{0,0}\right)+\ldots+\frac{\frac{\delta}{2}\left(1-\left(\frac{\delta}{2}\right)^{k}\right)}{1-\left(\frac{\delta}{2}\right)}\left(l_{r-1,1}-l_{r-1,0}\right)+\frac{\frac{\delta}{2}\left(1-\left(\frac{\delta}{2}\right)^{k}\right)}{1-\left(\frac{\delta}{2}\right)}\left(l_{r, 1}-l_{r, 0}\right) \\
& \leq l_{0,1}+\frac{\frac{\delta}{2}\left(1-\left(\frac{\delta}{2}\right)^{k}\right)}{1-\left(\frac{\delta}{2}\right)}\left(l_{0,1}-l_{0,0}\right)+\ldots \\
& +\frac{\frac{\delta}{2}\left(1-\left(\frac{\delta}{2}\right)^{k}\right)}{1-\left(\frac{\delta}{2}\right)}\left(\frac{\delta}{2}\right)^{k(r-1)}\left(l_{0,1}-l_{0,0}\right)+\frac{\frac{\delta}{2}\left(1-\left(\frac{\delta}{2}\right)^{k}\right)}{1-\left(\frac{\delta}{2}\right)}\left(\frac{\delta}{2}\right)^{k r}\left(l_{r, 1}-l_{r, 0}\right) \\
& =\left(l_{0,1}-l_{0,0}\right)\left[1+\frac{\frac{\delta}{2}\left(1-\left(\frac{\delta}{2}\right)^{k}\right)}{1-\frac{\delta}{2}} \sum_{s=0}^{r}\left(\frac{\delta}{2}\right)^{k s}\right]
\end{aligned}
$$

and $l_{r, i}$ is given for any $3 \leq i \leq k$, by

$$
\begin{aligned}
l_{r, i} & \leq l_{r, i-1}+\frac{\delta}{2}\left(l_{r, i-1}-l_{r, i-2}\right) \\
& \leq l_{r, i-2}+\frac{\delta}{2}\left(l_{r, i-2}-l_{r, i-3}\right)+\frac{\delta}{2}\left(l_{r, i-1}-l_{r, i-2}\right) \\
& \leq l_{r, i-2}+\frac{\delta}{2}\left(l_{r, i-2}-l_{r, i-3}\right)+\left(\frac{\delta}{2}\right)^{2}\left(l_{r, i-2}-l_{r, i-3}\right) \\
& \leq l_{r, 1}+\frac{\delta}{2}\left(\frac{1-\left(\frac{\delta}{2}\right)^{i-1}}{1-\frac{\delta}{2}}\right)\left(l_{r, 1}-l_{r, 0}\right) \\
& =\left(\frac{1-\left(\frac{\delta}{2}\right)^{i}}{1-\frac{\delta}{2}}\right)\left(l_{r, 1}-l_{r, 0}\right)+l_{r, 0} .
\end{aligned}
$$


This gives (32). Taking $i=k$ in (38), we get

$$
\begin{aligned}
l_{r, k}=l_{r+1,0} & =\left(\frac{1-\left(\frac{\delta}{2}\right)^{k}}{1-\frac{\delta}{2}}\right)\left[1+\left(\frac{\delta}{2}\right)^{k}+\ldots+\left(\frac{\delta}{2}\right)^{r k}\right]\left(l_{0,1}-l_{0,0}\right) \\
& =\left[\frac{1-\left(\frac{\delta}{2}\right)^{(r+1) k}}{1-\frac{\delta}{2}}\right]\left(l_{0,1}-l_{0,0}\right) .
\end{aligned}
$$

To show (34), it is sufficient to show that

$$
\begin{aligned}
& 0 \leq \frac{L}{2} \frac{\left(l_{r, 1}-l_{r, 0}\right)}{1-L_{0} l_{r, 0}} \leq \frac{\delta}{2} \\
& 0 \leq \frac{L}{2} \frac{\left(l_{r, 2}-l_{r, 0}\right)+\left(l_{r, 1}-l_{r, 0}\right)}{1-L_{0} l_{r, 0}} \leq \frac{\delta}{2} \\
& \ldots \ldots \\
& 0 \leq \frac{L}{2} \frac{\left(l_{r, k-2}-l_{r, 0}\right)+\left(l_{r, k-3}-l_{r, 0}\right)}{1-L_{0} l_{r, 0}} \leq \frac{\delta}{2} \\
& 0 \leq \frac{L}{2} \frac{\left(l_{r, k-1}-l_{r, 0}\right)+\left(l_{r, k-2}-l_{r, 0}\right)}{1-L_{0} l_{r, 0}} \leq \frac{\delta}{2} .
\end{aligned}
$$

Since $l_{r, k}$ is an increasing sequence, we can write

$\frac{L}{2} \frac{\left(l_{r, 1}-l_{r, 0}\right)}{1-L_{0} l_{r, 0}}<\frac{L}{2} \frac{\left(l_{r, 2}-l_{r, 0}\right)+\left(l_{r, 1}-l_{r, 0}\right)}{1-L_{0} l_{r, 0}}<\ldots<\frac{L}{2} \frac{\left(l_{r, k-1}-l_{r, 0}\right)+\left(l_{r, k-2}-l_{r, 0}\right)}{1-L_{0} l_{r, 0}}<\frac{L}{2} \frac{\left(l_{r, k}-l_{r, 0}\right)+\left(l_{r, k-1}-l_{r, 0}\right)}{1-L_{0} l_{r+1,0}}$.

Now, to show (40), it is sufficient to show that

$$
\frac{L}{2}\left(\frac{\left(l_{r, k}-l_{r, 0}\right)+\left(l_{r, k-1}-l_{r, 0}\right)}{1-L_{0} l_{r+1,0}}\right)<\frac{\delta}{2}
$$

(41) can be replaced by using (35), (36), (37), (38) and (39), by

$$
L\left(\frac{1-\left(\frac{\delta}{2}\right)^{k}}{1-\frac{\delta}{2}}+\frac{1-\left(\frac{\delta}{2}\right)^{k-1}}{1-\frac{\delta}{2}}\right)\left(\frac{\delta}{2}\right)^{r k} \eta+\delta L_{0} \frac{1-\left(\frac{\delta}{2}\right)^{(r+1) k}}{1-\frac{\delta}{2}}-\delta \leq 0 .
$$

Replacing $\frac{\delta}{2}$ by $u$ in (42), we introduce a recurrent function $f_{r, k}$ on $(0,1)$, defined by

$$
f_{r, k}(u)=L\left(\frac{1-u^{k}}{1-u}+\frac{1-u^{k-1}}{1-u}\right) u^{r k-1} \eta+2 L_{0} \eta \frac{1-u^{(r+1) k}}{1-u}-2
$$

To establish the relationship between two consecutive functions $f_{r, k}$, we replace $r$ by $r+1$ in (43). This gives

$$
\begin{aligned}
f_{r+1, k}(u) & =L\left(\frac{1-u^{k}}{1-u}+\frac{1-u^{k-1}}{1-u}\right) u^{(r+1) k-1} \eta+2 L_{0} \eta \frac{1-u^{(r+2) k}}{1-u}-2 \\
& =f_{r, k}(u)+L\left(\frac{1-u^{k}}{1-u}+\frac{1-u^{k-1}}{1-u}\right)\left(u^{(r+1) k-1}-u^{r k-1}\right) \eta+2 L_{0} \eta\left(\frac{1-u^{(r+2) k}}{1-u}-\frac{1-u^{(r+1) k}}{1-u}\right) \\
& =f_{r, k}(u)+u^{r k-1} \eta\left(u^{k}-1\right)\left(\frac{1-u^{k}}{1-u}+\frac{1-u^{k-1}}{1-u}\right)+2 u L_{0} \eta u^{(r+1) k} \frac{1-u^{k}}{1-u} \\
& =f_{r, k}(u)+u^{r k-1} \eta\left[L\left(u^{k}-1\right)\left(\frac{1-u^{k}}{1-u}+\frac{1-u^{k-1}}{1-u}\right)+2 u^{k+1} L_{0} \frac{1-u^{k}}{1-u}\right] \\
& =f_{r, k}(u)+H_{k}(u) u^{r k-1} \eta
\end{aligned}
$$


where $H_{k}(u)$ is defined in (29). Now, (42) is true, if

$$
f_{r, k} \leq 0 \text { for each } r=1,2, \ldots
$$

Define $f_{\infty, k}$ on $[0,1)$ by $f_{\infty, k}(u)=\lim _{r \rightarrow \infty} f_{r, k}(u)$. This gives $f_{\infty, k}(u)=\frac{2 L_{0} \eta}{1-u}-2 \leq 0$. By definition of $\alpha$ and using (44), we get $f_{\infty, k}(\alpha)=f_{r+1, k}(\alpha)=f_{r, k}(\alpha)$ for each $r$. Now, (45) is satisfied if $f_{\infty, k}(\alpha) \leq 0$. This holds true by (30). This implies that (34) is satisfied. Thus, $\left\{l_{n, k}\right\}$ is increasing, bounded above by $l^{* *}$ and from (39), it converges to the least upper bound $l^{*}$.

Theorem 2. Let $\mathscr{F}: \mathscr{D} \subset \mathscr{R}^{n} \rightarrow \mathscr{R}$ be a differentiable function such that the $\mathscr{B}$-condition and Lemma 2 hold true. Further assume that

1. There exists a point $x_{0}^{0} \in \mathscr{D}$, such that $\mathscr{F}\left(x_{0}^{0}\right) \neq 0, \nabla \mathscr{F}\left(x_{0}^{0}\right) \neq 0$.

2. $d_{0} \in \mathscr{R}^{n}$ such that $\left\|d_{0}\right\|=1$ and set

$$
\begin{aligned}
h_{0}^{0} & =-\frac{\mathscr{F}\left(x_{0}^{0}\right)}{\nabla \mathscr{F}\left(x_{0}^{0}\right) \cdot d_{0}} d_{0}, \quad h_{0}^{i}=-\frac{\mathscr{F}\left(x_{0}^{i}\right)}{\nabla \mathscr{F}\left(x_{0}^{i}\right) \cdot d_{0}} d_{0} \\
x_{0}^{i} & =x_{0}^{i-1}+h_{0}^{i-1}, \quad i \geq 1
\end{aligned}
$$

3. $\overline{\mathscr{B}}\left(x_{0}^{0}, l^{*}\right)=\left\{x \in \mathscr{R}^{n}:\left\|x-x_{0}^{0}\right\| \leq l^{*}\right\} \subset \mathscr{D}$, where $l^{*}=\lim _{n \rightarrow \infty} l_{n, i}$ and $\left\{l_{n, i}\right\}$ is given by (28).

4. $\left\{x_{n}^{i}\right\},(n \geq 0, i=1, \ldots, k)$ is generated by $x_{n}^{i}=x_{n}^{i-1}+h_{n}^{i-1}$ where $h_{n}^{i-1}=-\frac{\mathscr{F}\left(x_{n}^{i-1}\right)}{\nabla \mathscr{F}\left(x_{n}^{0}\right) \cdot d_{n}} d_{n}$ and $x_{n}^{k}=x_{n+1}^{0}$ satisfies $\angle\left(d_{n}, \nabla \mathscr{F}\left(x_{n}^{0}\right)\right) \leq \angle\left(d_{0}, \nabla \mathscr{F}\left(x_{0}^{0}\right)\right), n \geq 0$ for each $d_{n} \in \mathscr{R}^{n}$ such that $\left\|d_{n}\right\|=1$.

Then, starting with $x_{0}^{0}$, the sequence $\left\{x_{n}^{i}\right\}$ belongs to $\overline{\mathscr{B}}\left(x_{0}^{0}, l^{*}\right)$ for all $n \geq 0, i=1, \ldots, k$ and converges to a zero $x^{*} \in \overline{\mathscr{B}}\left(x_{0}^{0}, l^{*}\right)$ of (1). Moreover, $\nabla \mathscr{F}\left(x^{*}\right) \neq 0$ unless $\left\|x^{*}-x_{0}^{0}\right\|=l^{*}$ and $\nabla \mathscr{F}(x) \neq 0$ for all $x \in \overline{\mathscr{B}}\left(x_{0}^{0}, l^{*}\right)$. Furthermore, the following estimates hold for all $n \geq 0$ and $i=1, \ldots, k$.

$$
\begin{aligned}
\left\|x_{n+1}^{i}-x_{n}^{i}\right\| & \leq l_{n+1, i}-l_{n, i} \quad \text { and } \\
\left\|x_{n}^{i}-x^{*}\right\| & \leq l^{*}-l_{n, i}
\end{aligned}
$$

Proof. Using mathematical induction on $i$ and $n$, we first show that $\left\|x_{n}^{i}-x_{n}^{i-1}\right\| \leq l_{n, i}-l_{n, i-1}$. Under the assumptions of the Theorem 2 and $\mathscr{B}$-condition, it holds for $n=0$ and $i=1$ as

$$
\left\|x_{0}^{1}-x_{0}^{0}\right\|=\left\|h_{0}^{0}\right\| \leq \eta=l_{0,1}-l_{0,0}
$$

Now, for $n=0$ and $i=2$ and from Theorem 1 , we get

$$
\begin{aligned}
\left\|x_{0}^{2}-x_{0}^{1}\right\| & =\left\|h_{0}^{1}\right\|=\frac{\left|\mathscr{F}\left(x_{0}^{1}\right)\right|}{\left|\nabla \mathscr{F}\left(x_{0}^{0}\right) \cdot d_{0}\right|} \\
& \leq \frac{M}{2\left|\nabla \mathscr{F}\left(x_{0}^{0}\right) \cdot d_{0}\right|}\left\|x_{0}^{1}-x_{0}^{0}\right\|^{2} \\
& \leq \frac{L}{2}\left(l_{0,1}-l_{0,0}\right)^{2}=l_{0,2}-l_{0,1}
\end{aligned}
$$

and hence, we can get the inequality for $\mathscr{F}\left(x_{n}^{i}\right)$. Now, to get a consistent approximation for $\left\|\nabla \mathscr{F}\left(x_{n}^{0}\right)\right\|^{-1}$, we have

$$
\begin{aligned}
\left\|\nabla \mathscr{F}\left(x_{0}^{0}\right)\right\| & =\left\|\nabla \mathscr{F}\left(x_{0}^{0}\right)-\nabla \mathscr{F}\left(x_{n}^{0}\right)+\nabla \mathscr{F}\left(x_{n}^{0}\right)\right\| \\
& \leq\left\|\nabla \mathscr{F}\left(x_{n}^{0}\right)\right\|+\left\|\nabla \mathscr{F}\left(x_{n}^{0}\right)-\nabla \mathscr{F}\left(x_{0}^{0}\right)\right\|
\end{aligned}
$$


This gives,

$$
\begin{aligned}
\left\|\nabla \mathscr{F}\left(x_{n}^{0}\right)\right\| & \geq\left\|\nabla \mathscr{F}\left(x_{0}^{0}\right)\right\|-\left\|\nabla \mathscr{F}\left(x_{n}^{0}\right)-\nabla \mathscr{F}\left(x_{0}^{0}\right)\right\| \\
& \geq\left\|\nabla \mathscr{F}\left(x_{0}^{0}\right)\right\|\left(1-\left\|\nabla \mathscr{F}\left(x_{0}^{0}\right)\right\|^{-1} M_{0}\left\|x_{n}^{0}-x_{0}^{0}\right\|\right) \\
& =\left\|\nabla \mathscr{F}\left(x_{0}^{0}\right)\right\|\left(1-L_{0}\left\|x_{n}^{0}-x_{0}^{0}\right\|\right) \\
& \geq\left\|\nabla \mathscr{F}\left(x_{0}^{0}\right)\right\|\left(1-L_{0} l_{n, 0}\right) .
\end{aligned}
$$

This gives

$$
\left\|\nabla \mathscr{F}\left(x_{n}^{0}\right)\right\|^{-1} \leq \frac{\left\|\nabla \mathscr{F}\left(x_{0}^{0}\right)\right\|^{-1}}{1-L_{0} l_{n, 0}} \forall n \geq 0 .
$$

Now, using (5) and (48), we get

$$
\frac{1}{\left|\nabla \mathscr{F}\left(x_{n}^{0}\right) \cdot d_{n}\right|} \leq \frac{\left\|\nabla \mathscr{F}\left(x_{0}^{0}\right)\right\|}{\| \nabla \mathscr{F}\left(x_{n}^{0}\right)||\left|\nabla \mathscr{F}\left(x_{0}^{0}\right) \cdot d_{0}\right|} \leq \frac{\left|\nabla \mathscr{F}\left(x_{0}^{0}\right) \cdot d_{0}\right|^{-1}}{1-L_{0} l_{n, 0}} .
$$

Using (49) and derivation of $\mathscr{F}\left(x_{n}^{i}\right)$ as is done in Theorem 1, we can show (46) for $i=1$, by

$$
\begin{aligned}
\left\|x_{n}^{i+1}-x_{n}^{i}\right\| & =\left\|h_{n}^{i}\right\|=\frac{\left|\mathscr{F}\left(x_{n}^{i}\right)\right|}{\left|\nabla \mathscr{F}\left(x_{n}^{0}\right) \cdot d_{n}\right|} \\
& \leq \frac{M}{2} \frac{\left|\nabla \mathscr{F}\left(x_{0}^{0}\right) \cdot d_{0}\right|^{-1}}{1-L_{0} l_{n, 0}}\left\|x_{n}^{i}-x_{n}^{i-1}\right\|^{2} \\
& \leq \frac{L}{2} \frac{\left(l_{n, i}-l_{n, i-1}\right)^{2}}{1-L_{0} l_{n, 0}}=l_{n, i+1}-l_{n, i}
\end{aligned}
$$

and for $i \geq 2$, by

$$
\begin{aligned}
\left\|x_{n}^{i+1}-x_{n}^{i}\right\| & =\left\|h_{n}^{i}\right\|=\frac{\left|\mathscr{F}\left(x_{n}^{i}\right)\right|}{\left|\nabla \mathscr{F}\left(x_{n}^{0}\right) \cdot d_{n}\right|} \\
& \leq \frac{M}{2} \frac{\left|\nabla \mathscr{F}\left(x_{0}^{0}\right) \cdot d_{0}\right|^{-1}}{1-L_{0} l_{n, 0}}\left(\left\|x_{n}^{i}-x_{n}^{0}\right\|+\left\|x_{n}^{i-1}-x_{n}^{0}\right\|\right)\left(x_{n}^{i}-x_{n}^{i-1}\right) \\
& \leq \frac{L}{2} \frac{\left(l_{n, i}-l_{n, 0}\right)+\left(l_{n, i-1}-l_{n, 0}\right)}{1-L_{0} l_{n, 0}}\left(l_{n, i}-l_{n, i-1}\right)=l_{n, i+1}-l_{n, i} .
\end{aligned}
$$

Combining (50) and (51), we get (46). Moreover, for each $\gamma \in \overline{\mathscr{B}}\left(x_{n}^{i+1}, l^{*}-l_{n, i+1}\right)$, we get

$$
\left\|\gamma-x_{n}^{i}\right\| \leq\left\|\gamma-x_{n}^{i+1}\right\|+\left\|x_{n}^{i+1}-x_{n}^{i}\right\| \leq l^{*}-l_{n, i+1}+l_{n, i+1}-l_{n, i}=l^{*}-l_{n, i}
$$

This gives (47) for all $n \geq 0$. Lemma 2 guarantees that $\left\{l_{n, i}\right\}$ is a Cauchy sequence. It follows from (46) and (47) that $\left\{x_{n}^{i}\right\}$ is a Cauchy sequence and it converge to some $x^{*} \in \overline{\mathscr{B}}\left(x_{0}, l^{*}\right)$. Now, we have to show that $x^{*}$ is a solution of (1). Using Theorem 1 , we get for $i \neq 1$

$$
\left|\mathscr{F}\left(x_{n}^{i}\right)\right| \leq \frac{M}{2}\left[\left(l_{n, i}-l_{n, 0}\right)+\left(t_{n, i-1}-l_{n, 0}\right)\right]\left(l_{n, i}-l_{n, i-1}\right) \rightarrow 0 \text { as } n \rightarrow \infty .
$$

and for $i=1$,

$$
\left|\mathscr{F}\left(x_{n}^{i}\right)\right| \leq \frac{M}{2}\left(l_{n, i}-l_{n, 0}\right)^{2} \rightarrow 0 \text { as } n \rightarrow \infty .
$$


From (52), (53) and continuity of $\mathscr{F}$, we conclude that $x^{*}$ is a solution of (1). Now, we shall show that $\nabla \mathscr{F}(x) \neq 0$ for all $x \in \overline{\mathscr{B}}\left(x_{0}, l^{*}\right)$. Using the definition of $L_{0}$ and Lemma 2 , we have

$$
\left\|\nabla \mathscr{F}(x)-\nabla \mathscr{F}\left(x_{0}^{0}\right)\right\| \leq M_{0}\left\|x-x_{0}\right\| \leq M_{0} l^{*} \leq\left\|\nabla \mathscr{F}\left(x_{0}^{0}\right)\right\|
$$

If $\left\|x-x_{0}^{0}\right\|<l^{*}$, we get

$$
\left\|\nabla \mathscr{F}(x)-\nabla \mathscr{F}\left(x_{0}^{0}\right)\right\|<M_{0} l^{*}<\left\|\nabla \mathscr{F}\left(x_{0}^{0}\right)\right\|
$$

or

$$
\left\|\nabla \mathscr{F}\left(x_{0}^{0}\right)\right\|>\left\|\nabla \mathscr{F}(x)-\nabla \mathscr{F}\left(x_{0}^{0}\right)\right\|
$$

This shows that $\nabla \mathscr{F}(x) \neq 0$. Thus, the proof of Theorem 2 is completed.

Remarks 1. The limits points $l^{*}$ in Theorem 2 can be replaced by $l^{* *}$.

Corollary 1. Let $\mathscr{F}, x_{0}, M_{0}, M, \eta, L_{0}$, L are same as in Theorems 1 and 2. Define direction $d_{n}$ and step $h_{n}^{0}$ for all $n \geq 0$ and $i=1,2, \ldots, k$, by

$$
d_{n}=\frac{\nabla \mathscr{F}\left(x_{n}^{0}\right)}{\left\|\nabla \mathscr{F}\left(x_{n}^{0}\right)\right\|} \text { and } h_{n}^{i}=-\frac{\mathscr{F}\left(x_{n}^{i}\right)}{\left\|\nabla \mathscr{F}\left(x_{n}^{0}\right)\right\|^{2}} \nabla \mathscr{F}\left(x_{n}^{0}\right)
$$

Then starting with $x_{0}^{0}$ and for $x_{n+1}^{0}=x_{n}^{k}$, the gradient method given by

$$
\begin{aligned}
x_{n}^{1}= & x_{n}^{0}-\frac{\mathscr{F}\left(x_{n}^{0}\right)}{\left\|\nabla \mathscr{F}\left(x_{n}^{0}\right)\right\|^{2}} \nabla \mathscr{F}\left(x_{n}^{0}\right) \\
x_{n}^{2}= & x_{n}^{1}-\frac{\mathscr{F}\left(x_{n}^{1}\right)}{\left\|\nabla \mathscr{F}\left(x_{n}^{0}\right)\right\|^{2}} \nabla \mathscr{F}\left(x_{n}^{0}\right) \\
& \ldots \ldots \ldots \cdots \cdots \\
x_{n}^{k}= & x_{n}^{k-1}-\frac{\mathscr{F}\left(x_{n}^{k-1}\right)}{\left\|\nabla \mathscr{F}\left(x_{n}^{0}\right)\right\|^{2}} \nabla \mathscr{F}\left(x_{n}^{0}\right)
\end{aligned}
$$

satisfies the Conclusions of Theorems 1 and 2. This implies that the gradient method [1, 4, 9] comes out as a special case of our work.

Corollary 2. If we choose the direction $d_{n}$ as the unit vector along the maximal modulus $e^{m(n)}$, where $m(n)$ is the index of the component of $\nabla \mathscr{F}\left(x_{n}\right)$ of maximal modulus given by

$$
\left|\nabla \mathscr{F}\left(x_{n}^{0}\right)[m(n)]\right|:=\max _{l=1,2, \ldots, n}\left|\nabla \mathscr{F}\left(x_{n}^{0}\right)[l]\right|
$$

Then for this choice of $d_{n}$, (4) becomes

$$
\begin{aligned}
x_{n}^{1}= & x_{n}^{0}-\frac{\mathscr{F}\left(x_{n}^{0}\right)}{\nabla \mathscr{F}\left(x_{n}^{0}\right)[m(n)]} e^{m(n)} \\
x_{n}^{2}= & x_{n}^{1}-\frac{\mathscr{F}\left(x_{n}^{1}\right)}{\nabla \mathscr{F}\left(x_{n}^{0}\right)[m(n)]} e^{m(n)} \\
& \cdots \cdots \cdots \cdots \cdot \\
x_{n}^{k}= & x_{n}^{k-1}-\frac{\mathscr{F}\left(x_{n}^{k-1}\right)}{\nabla \mathscr{F}\left(x_{n}^{0}\right)[m(n)]} e^{m(n)}
\end{aligned}
$$

Theorems 1 and 2 can be applied if $\left|\nabla \mathscr{F}\left(x_{n}^{0}\right) \cdot d_{n}\right|$ is replaced by $\left\|\nabla \mathscr{F}\left(x_{n}^{0}\right)\right\|_{\infty}$ in each occurrence using $\infty$-norm instead of Euclidean norm. An analog of Theorem 2 is given by 
Theorem 3. Let $\mathscr{F}: \mathscr{D} \subset \mathscr{R}^{n} \rightarrow \mathscr{R}$ be a differentiable function. If $x_{0}^{0} \in \mathscr{D}$ is such that $\mathscr{F}\left(x_{0}^{0}\right) \neq 0$ and $\nabla \mathscr{F}\left(x_{0}^{0}\right) \neq 0$. Assume

$$
\begin{aligned}
\left|\mathscr{F}\left(x_{0}^{0}\right)\right| & \leq\left|\nabla \mathscr{F}\left(x_{0}^{0}\right)[m(n)]\right| \eta \\
\|\nabla \mathscr{F}(x)-\nabla \mathscr{F}(y)\|_{\infty} & \leq M\|x-y\|_{\infty} \\
\left\|\nabla \mathscr{F}(x)-\nabla \mathscr{F}\left(x_{0}\right)\right\|_{\infty} & \leq M_{0}\left\|x-x_{0}\right\|_{\infty} \\
L & =M\left|\nabla \mathscr{F}\left(x_{0}^{0}\right)[m(n)]\right|^{-1} \\
L_{0} & =M_{0}\left|\nabla \mathscr{F}\left(x_{0}^{0}\right)[m(n)]\right|^{-1}
\end{aligned}
$$

and $\overline{\mathscr{B}}\left(x_{0}, l^{*}\right) \subset \mathscr{D}$. Then the sequence defined by (54) is well defined, belongs to $\overline{\mathscr{B}}\left(x_{0}, l^{*}\right)$ and converges to a solution $x^{*} \in \overline{\mathscr{B}}\left(x_{0}, l^{*}\right)$. Moreover, $\nabla \mathscr{F}\left(x^{*}\right) \neq 0$ unless $\left\|x^{*}-x_{0}\right\|_{\infty}=l^{*}$ and $\nabla \mathscr{F}(x) \neq 0$ for all $x \in \overline{\mathscr{B}}\left(x_{0}, l^{*}\right)$. Furthermore, the following estimates hold for all $n \geq 0$ and $i=1, \ldots, k$.

$$
\begin{aligned}
\left\|x_{n+1}^{i}-x_{n}^{i}\right\|_{\infty} & \leq l_{n+1, i}-l_{n, i} \quad \text { and } \\
\left\|x_{n}^{i}-x^{*}\right\|_{\infty} & \leq l^{*}-l_{n, i}
\end{aligned}
$$

Theorem 3 can be proved in a similar way as Theorem 2 is proved. Thus it is also a special case of our work.

Corollary 3. This work can be extended in Hilbert space setting. Here, $\mathscr{F}$ is a differential operator defined on a convex subset $\mathscr{D}$ of a Hilbert space $H$ with values in $\mathscr{R}$. Only, some properties of inner product and conditions on angle are changed. We take $\|x\|=\sqrt{\langle x \cdot x\rangle}$. The condition $\angle\left(d_{n}, \nabla \mathscr{F}\left(x_{n}^{0}\right)\right) \leq \angle\left(d_{0}, \nabla \mathscr{F}\left(x_{0}^{0}\right)\right)$ is relaxed by using $\left|\nabla \mathscr{F}\left(x_{n}^{0}\right) \cdot d_{n}\right| \geq \lambda\left\|\nabla \mathscr{F}\left(x_{n}^{0}\right)\right\|$ where $\left\|d_{n}\right\|=1$ and $\lambda \in[0,1]$ as one can always choose $\lambda=\frac{\left|\nabla \mathscr{F}\left(x_{n}^{0}\right) \cdot d_{n}\right|}{\left\|\nabla \mathscr{F}\left(x_{0}^{0}\right)\right\|} \leq 1$. For using Theorems 1 and 2 , we set

$$
L_{0}=\frac{M_{0}}{\lambda\left\|\nabla \mathscr{F}\left(x_{0}^{0}\right)\right\|}, L=\frac{M}{\lambda\left\|\nabla \mathscr{F}\left(x_{0}^{0}\right)\right\|} \text { and } \eta=\frac{\left|\mathscr{F}\left(x_{0}^{0}\right)\right|}{\lambda\left\|\nabla \mathscr{F}\left(x_{0}^{0}\right)\right\|}
$$

Corollary 4. This work can also be extended to k-step Newton's method with frozen first derivative [6, 17] in Banach-spaces setting.

\section{Numerical Examples}

In this section, two numerical examples are worked out to show the applicability of (4). The Euclidean inner product and the corresponding vectors and matrices norms are used. The high level software MATLAB R2012b on an Intel(R) core (TM) i5-3470 CPU 3.20 GHz with 4GB of RAM running on the windows 7 Professional version 2009 Service Pack 1 is used.

Example 1. Consider the cubic polynomial equation $\mathscr{F}$ in two dimensions on $\mathscr{D}$ given in [5, 9, 11], by

$$
\mathscr{F}(x)=\frac{\gamma_{1}^{3}+\gamma_{2}^{3}}{2}-\theta, x=\left(\gamma_{1}, \gamma_{2}\right)^{T}
$$

Choose $x_{0}=(1,1)^{T}$ and $\mathscr{D}=\left\{x:\left\|x-x_{0}\right\| \leq 1-\theta\right\}$ for $\theta \in[0,1)$. The gradient $\nabla \mathscr{F}$ of $\mathscr{F}$ is given by

$$
\nabla \mathscr{F}(x)=\frac{3}{2}\left(\gamma_{1}^{2}, \gamma_{2}^{2}\right)^{T}
$$


This gives $M=3 \sqrt{2}(2-\theta), M_{0}=\frac{3 \sqrt{2}}{2}(3-\theta), \eta=\frac{\sqrt{2}}{3}(1-\theta)$ and $\beta=\frac{\sqrt{2}}{3}$. For $\theta=0.6166$, we get $L=2.7668$ and $L_{0}=2.3834$. Theorem 1 requires $R$ to satisfy $R=\frac{1+\frac{a_{0}}{2} \frac{1-s_{0}^{k-1}}{1-s_{0}}}{1-\frac{s_{0}^{k-1} a_{0}}{2}}$. For $k=1$, we get $R=$ $\frac{1+\frac{a_{0}}{2\left(1-s_{0}\right)}}{1-\frac{a_{0}}{2}}$. This implies that there exists a real solution of the polynomial

$\left(2 M_{0} \beta \eta+2 M_{0}^{2} \beta^{2} \eta^{2}\right) R^{3}+\left(L^{2} \eta^{2}-M_{0} L \beta \eta^{2}-4 M_{0} \beta \eta-2 M_{0}^{2} \beta^{2} \eta^{2}-2\right) R^{2}+\left(L \eta+5 M_{0} \beta \eta+2\right) R-3=0$.

On substituting the expressions for $a_{0}$ and $s_{0}$ from Theorem 1 and simplifying. This gives $R=2.02108$ and $M_{0} \beta R \eta=0.8706<1$ but $\frac{a_{0}}{2}=1.9325>1$. Hence, Theorem 1 is not applicable for (4). Now, we verify the conditions of Theorem 2. For $k=1$ and $H_{1}(t)=2 L_{0} t^{2}+L t-L$, we get $\alpha=0.5250, \delta=2 \alpha=1.050$. Also, $\frac{L \eta}{1-L_{0} \eta}=0.8785<\delta=1.0501<2\left(1-L_{0} \eta\right)=1.1138$ and $l^{* *}=0.3805$. Hence, all the conditions of Theorem 2 are satisfied and (4) is applicable.

Example 2. Consider a nonlinear integral equation of mixed Hammerstein type given by

$$
x(s)=2+\frac{1}{3} \int_{0}^{1} G(s, t) x(t)^{2} d t
$$

where $x \in \mathscr{C}[0,1], s \in[0,1]$ such that $\|x\| \leq 2$ and $G(s, t)$ is the Green's function given by

$$
G(s, t)= \begin{cases}(1-s) t, & t \leq s \\ (1-t) s, & s \leq t .\end{cases}
$$

Solving (55) is equivalent to solving $\mathscr{F}(x)=0$, where

$$
\mathscr{F}(x)=x(s)-2-\frac{1}{3} \int_{0}^{1} G(s, t) x(t)^{2} d t .
$$

We shall solve (56) after transforming it to a finite dimensional problem. For this, we use Gauss -Legendre formula [20]

$$
\int_{0}^{1} v(t) d t=\sum_{j=1}^{m} \beta_{j} v\left(t_{j}\right)
$$

where $\beta_{j}$ and $t_{j}$ are weights and nodes given in Table 1 for $m=8$. We denote the approximation of $x\left(t_{j}\right)$ by $x_{j},(j=1, \ldots, m)$. Now, (55) is equivalent to the nonlinear system of equations given by

Table 1: Weights and Nodes in Gauss-Legendre Formula

\begin{tabular}{ccccccccc}
\hline $\mathrm{j}$ & 1 & 2 & 3 & 4 & 5 & 6 & 7 & 8 \\
\hline Weight $\left(\beta_{j}\right)$ & 0.050614 & 0.111190 & 0.156853 & 0.181341 & 0.181341 & 0.156853 & 0.111190 & 0.050614 \\
\hline Nodes $\left(t_{j}\right)$ & 0.019855 & 0.101666 & 0.237233 & 0.408282 & 0.591717 & 0.762766 & 0.898333 & 0.980144 \\
\hline
\end{tabular}

$$
x_{i}-2-\frac{1}{3} \sum_{j=1}^{8} a_{i j} x_{j}^{2}=0, \quad i=1,2, \ldots, 8
$$

where,

$$
a_{i j}= \begin{cases}\beta_{j} t_{j}\left(t_{i}-1\right) / 2, & \text { if } j \leq i, \\ \beta_{j} t_{i}\left(t_{j}-1\right) / 2, & \text { if } i<j .\end{cases}
$$


(57) can be expressed as

$$
f_{i}(x)=x_{i}-2-\frac{1}{3} \sum_{j=1}^{m} a_{i j} x_{j}^{2}, \quad i=1,2, \ldots, 8 .
$$

To solve (57) with (4), we first transform it into $\mathscr{F}: \mathscr{R}^{n} \rightarrow \mathscr{R}$, by

$$
\mathscr{F}(x)=\sum_{i=1}^{i=8} f_{i}(x)^{2}
$$

We take $M=\sup _{x}\left\|\mathscr{F}^{\prime \prime}(x)\right\|$. Starting with $x_{0}=(1,1, \ldots, 1)$, we get $\beta=0.1663, \eta=1.1460, M=$

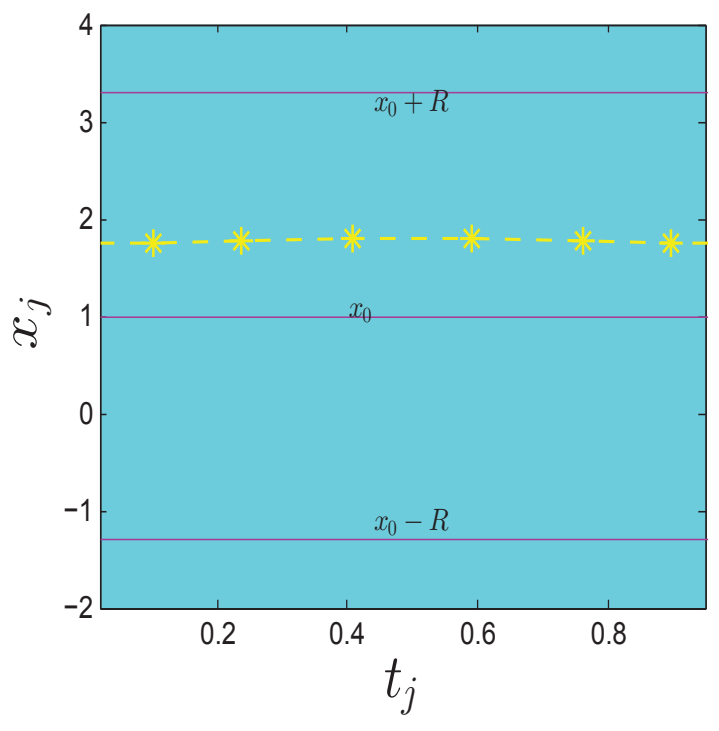

Figure 1: Approximate solution of (57).

2.3508, $M_{0}=2.3508, L=L_{0}=0.3910$. We use the direction vector $d_{n}=\frac{\nabla \mathscr{F}\left(x_{n}^{0}\right)}{\left\|\nabla \mathscr{F}\left(x_{n}^{0}\right)\right\|}$. It is easy to see that all the conditions of recurrent functions approach is satisfied as $\frac{L \eta}{1-L_{0} \eta}=0.8119<\delta=1<$ $2\left(1-L_{0} \eta\right)=1.1038$. So, Theorem 2 is applicable for this problem and we get the solution (1.7408..., $1.7604 \ldots, 1.7849 \ldots, 1.8017 \ldots, 1.8017 \ldots, 1.7849 \ldots, 1.7603 \ldots, 1.7408 \ldots$.$) . For k=1$, it gives $\alpha=0.5$ and $l^{* *}=\eta /(1-\alpha)=2.29207$. The approximate solution can be visualized in Fig. 1 . Where, it represents the plot $\left(t_{j}, x_{j}\right)$ for $j=1,2,3, \ldots, 8$. But, when we look for the solution by recurrent relations approach, we get $R=2.1551, M_{0} \beta R \eta=0.9657<1$ but $\frac{a_{0}}{2}=6.5278>1$. Thus, we can not apply Theorem 1 .

\section{Computational order of convergence and computational efficiency}

In this section, the computational order of convergence $\operatorname{COC}(\sigma)[11,15,16]$ and the computational efficiency $(C E)$ for different steps of (4) are compared. They are defined as

$$
\sigma=\frac{\ln \left(\left\|x_{n}^{k}-x^{*}\right\| /\left\|x_{n-1}^{k}-x^{*}\right\|\right)}{\ln \left(\left\|x_{n-1}^{k}-x^{*}\right\| /\left\|x_{n-2}^{k}-x^{*}\right\|\right)} \text { and } C E=\sigma^{1 /(O C * I N)},
$$


where $O C$ is the operational cost per iteration and $I N$ is the total iteration number. In this case, if the operator $\mathscr{F}$ is such that $\mathscr{F}: \mathscr{D} \subseteq \mathscr{R}^{n} \rightarrow \mathscr{R}$ then $2 n+1$ is the operational cost to perform (2) (k=1). So, the total operational cost per iteration for $(4)$ is $n+(n+1) k$. To compute the computational order of convergence $(\sigma)$ and the computational efficiency $(C E)$, we consider the nonlinear problem considered in [11].

\section{Example 3.}

$$
\mathscr{F}(x)=\sum_{i=1}^{p}\left(\sin x_{i}\right)^{2}+\sum_{i=p+1}^{m}\left(\tan x_{i}\right)^{2}, \mathrm{p} \text { is a given integer. }
$$

Here, we consider nonlinear systems of different sizes. From the starting point $x_{0}^{0}=(0.1,0.1, \ldots, 0.1)$, the computational order of convergence, the computational efficiency and the iteration number denoted by COC, CE and IN, respectively are given for different values of $m$ and $p$ in Tables 2, 3 and 4 in logarithmic scale. The stopping criteria $\left|\mathscr{F}\left(x_{n}^{0}\right)\right|<10^{-5}$ is used here. We have considered (4) for different step using $k=1,2, \ldots, 10$. The direction $d_{n}$ is chosen in such a way that it is sufficiently close to the gradient vector $\nabla \mathscr{F}\left(x_{n}^{0}\right)$. It can be seen that if $\nabla \mathscr{F}\left(x_{n}^{0}\right) \neq 0$, then the vector $d_{n}=\frac{\nabla \mathscr{F}\left(x_{n}^{0}\right)}{\left\|\nabla \mathscr{F}\left(x_{n}^{0}\right)\right\|}$ is the unit vector near to $\nabla \mathscr{F}\left(x_{n}^{0}\right)$. Here, we have used this direction.

Table 2: $C O C, C E$ and $I N$ for $(m=10, p=5)$ and $(m=20, p=5)$

\begin{tabular}{|c|c|c|c|c|c|c|c|c|c|c|c|}
\hline$m$ & $p$ & $k$ & $\operatorname{COC}(\sigma)$ & $C E$ & $I N$ & $m$ & $p$ & $k$ & $\operatorname{COC}(\sigma)$ & $C E$ & $I N$ \\
\hline \multirow[t]{10}{*}{10} & 5 & 1 & 0.000923719 & $1.46622 \mathrm{E}-05$ & 19 & 20 & 5 & 1 & 0.002057358 & $1.67265 \mathrm{E}-05$ & 19 \\
\hline & & 2 & 0.000952065 & $9.91735 \mathrm{E}-06$ & 16 & & & 2 & 0.002170597 & 1.16699E-05 & 16 \\
\hline & & 3 & 0.000952698 & $7.38526 \mathrm{E}-06$ & 14 & & & 3 & 0.002200687 & 8.8381E-06 & 15 \\
\hline & & 4 & 0.000944839 & 5.83234E-06 & 13 & & & 4 & 0.002201381 & 7.05571E-06 & 13 \\
\hline & & 5 & 0.000934072 & 4.79011E-06 & 12 & & & 5 & 0.002189731 & $5.83928 \mathrm{E}-06$ & 13 \\
\hline & & 6 & 0.000922447 & $3.48969 \mathrm{E}-06$ & 12 & & & 6 & 0.002172578 & 5.79354E-06 & 12 \\
\hline & & 7 & 0.000910809 & 7.06053E-06 & 11 & & & 7 & 0.002153048 & 4.2975E-06 & 11 \\
\hline & & 8 & 0.000899514 & $3.05957 \mathrm{E}-06$ & 11 & & & 8 & 0.002132685 & $3.78136 \mathrm{E}-06$ & 11 \\
\hline & & 9 & 0.000889524 & $2.71778 \mathrm{E}-06$ & 10 & & & 9 & 0.002112281 & $3.36887 \mathrm{E}-06$ & 11 \\
\hline & & 10 & 0.000878454 & $2.44015 \mathrm{E}-06$ & 10 & & & 10 & 0.002092255 & $3.03225 \mathrm{E}-06$ & 10 \\
\hline
\end{tabular}

It can be observed from Tables 2, 3 and 4 that the computational efficiency obtained by different steps are competitive to each other. It can be observed that COC increases with increase of steps. After fourth step, COC is being decreased. Thus, it can also be concluded that the most efficient (4) are obtained with $k=2,3,4$.

\section{Conclusions}

The directional k-step Newton methods for a finite positive integer $k$ is developed for solving a single nonlinear equation in $n$ variables. Using a combination of Lipschitz and center-Lipschitz continuity conditions, its semilocal convergence analysis is established from two different approaches. The first approach is based on recurrent relations whereas the second is based on recurrent functions. It is found that the second approach is more favorable as problems can be constructed for which its sufficient conditions are satisfied but failed to satisfy the sufficient conditions given by first one. The convergence theorems 
Table 3: COC, $C E$ and $I N$ for $(m=20, p=10)$ and $(m=50, p=15)$

\begin{tabular}{|c|c|c|c|c|c|c|c|c|c|c|c|}
\hline$m$ & $p$ & $k$ & $\operatorname{COC}(\sigma)$ & $C E$ & $I N$ & $m$ & $p$ & $k$ & $\operatorname{COC}(\sigma)$ & $C E$ & $I N$ \\
\hline \multirow[t]{10}{*}{20} & 10 & 1 & 0.000923719 & $7.50991 \mathrm{E}-06$ & 19 & 50 & 15 & 1 & 0.001836645 & $6.06153 \mathrm{E}-06$ & 20 \\
\hline & & 2 & 0.000952065 & $5.11863 \mathrm{E}-06$ & 16 & & & 2 & 0.001931912 & 4.23665E-06 & 17 \\
\hline & & 3 & 0.000952698 & $3.8261 \mathrm{E}-06$ & 15 & & & 3 & 0.001955418 & $3.21087 \mathrm{E}-06$ & 15 \\
\hline & & 4 & 0.000944839 & $3.02833 \mathrm{E}-06$ & 13 & & & 4 & 0.001953899 & $2.56417 \mathrm{E}-06$ & 14 \\
\hline & & 5 & 0.000934072 & $2.49086 \mathrm{E}-06$ & 13 & & & 5 & 0.001942045 & $2.12245 \mathrm{E}-06$ & 13 \\
\hline & & 6 & 0.000922447 & $2.10604 \mathrm{E}-06$ & 12 & & & 6 & 0.0019257 & $1.80309 \mathrm{E}-06$ & 12 \\
\hline & & 7 & 0.000910809 & $1.81798 \mathrm{E}-06$ & 11 & & & 7 & 0.001907508 & $1.56225 \mathrm{E}-06$ & 12 \\
\hline & & 8 & 0.000901658 & $1.59488 \mathrm{E}-06$ & 11 & & & 8 & 0.001888759 & $1.37464 \mathrm{E}-06$ & 11 \\
\hline & & 9 & 0.000888713 & $1.4174 \mathrm{E}-06$ & 11 & & & 9 & 0.00187011 & $1.2247 \mathrm{E}-06$ & 11 \\
\hline & & 10 & 0.000878454 & $1.27312 \mathrm{E}-06$ & 10 & & & 10 & 0.001851896 & $1.10232 \mathrm{E}-06$ & 11 \\
\hline
\end{tabular}

Table 4: $C O C, C E$ and $I N$ for $(m=80, p=40)$ and $(m=200, p=50)$

\begin{tabular}{|c|c|c|c|c|c|c|c|c|c|c|c|}
\hline$m$ & $p$ & $k$ & $\operatorname{COC}(\sigma)$ & $C E$ & IN & $m$ & $p$ & $k$ & $\operatorname{COC}(\sigma)$ & $C E$ & $I N$ \\
\hline \multirow[t]{10}{*}{80} & 40 & 1 & 0.000923719 & $1.91246 \mathrm{E}-06$ & 20 & 200 & 50 & 1 & 0.002057358 & $1.71019 \mathrm{E}-06$ & 21 \\
\hline & & 2 & 0.000952065 & $1.31138 \mathrm{E}-06$ & 17 & & & 2 & 0.002170597 & $1.20188 \mathrm{E}-06$ & 17 \\
\hline & & 3 & 0.000952698 & $9.83177 \mathrm{E}-07$ & 15 & & & 3 & 0.002200687 & $9.13527 \mathrm{E}-07$ & 16 \\
\hline & & 4 & 0.000944839 & 7.7957E-07 & 14 & & & 4 & 0.002201381 & 7.3087E-07 & 14 \\
\hline & & 5 & 0.000934072 & 6.41974E-07 & 13 & & & 5 & 0.002189731 & $6.05735 \mathrm{E}-07$ & 14 \\
\hline & & 6 & 0.000922447 & $5.43255 \mathrm{E}-07$ & 13 & & & 6 & 0.002172578 & $5.15073 \mathrm{E}-07$ & 13 \\
\hline & & 7 & 0.000910809 & 4.69247E-07 & 12 & & & 7 & 0.002153048 & $4.46598 \mathrm{E}-07$ & 12 \\
\hline & & 8 & 0.000899514 & $4.11865 \mathrm{E}-07$ & 11 & & & 8 & 0.002132685 & 4.42374E-07 & 12 \\
\hline & & 9 & 0.000888713 & $3.66177 \mathrm{E}-07$ & 11 & & & 9 & 0.002112281 & $3.5047 \mathrm{E}-07$ & 11 \\
\hline & & 10 & 0.000878454 & $3.29009 \mathrm{E}-07$ & 11 & & & 10 & 0.002092255 & $3.15574 \mathrm{E}-07$ & 11 \\
\hline
\end{tabular}

for the existence and uniqueness of the solution for each of them are established. Numerical examples including nonlinear Hammerstein type integral equations are worked out and significantly improved results are obtained. Further, the computational order of convergence and the computational efficiency are derived in order to find the suitable $k$.

\section{References}

[1] Y. Levin, B. Israel. Directional Newton Methods in n Variables. Math. Comp. A.M.S. 71(237)(2002): 251-262.

[2] G. Lukács. The generalized inverse matrix and the surface surface intersection problem. The theory and practice of geometric modeling. Springer Berlin Heidelberg, 1989. 167-185.

[3] I. K. Argyros and Ángel Alberto Magreñán. Extending the applicability of Gauss-Newton method for convex composite optimization on Riemannian manifolds. Appl. Math. Comput. 249 (2014): 453-467. 
[4] I. K. Argyros. A semilocal convergence analysis for directional Newton methods. Math. Comput. A.M.S. 80(2011): 327-343.

[5] I. K. Argyros, S. Hilout. A convergence analysis for directional two-step Newton methods. Numer. Algor. 55 (2010): 503-528.

[6] S. Amat, C. Bermúdez, M. A. Hernández-Verón, E. Martínez. On an efficient k-step iterative method for nonlinear equations. J. Comput. Appl. Math. 302 (2016): 258-271.

[7] P. K. Parida, D. K. Gupta. Recurrence relations for semilocal convergence of a Newton-like method in Banach spaces. J. Math. Anal. Appl. 345(2008): 350-361.

[8] I. K. Argyros, S. George. Local convergence for some high convergence order Newton-like methods with frozen derivatives. SeMA 70(2015): 47-59.

[9] I. K. Argyros, S. Hilout. Convergnce of Directional Methods under mild differentiability and applications. Appl. Math. Comput. 217(2011) : 8731- 8746.

[10] E. Martínez, S. Singh, J. L. Hueso, D. K. Gupta. Enlarging the convergence domain in local convergence studies for iterative methods in Banach spaces. Appl. Math. Comput. 281(2016), 252-265.

[11] I. K. Argyros, M. A. Henandez, S. Hilout, N. Romero. Directional Chebyshev-type methods for solving equations. Math. Comput. A.M.S. 84(2015): 815-830.

[12] S. K. Parhi, D. K. Gupta. Convergence of Stirling's method under weak differentiability condition. Mathematical Methods in the Applied Sciences 34(2011): 168-175.

[13] I. K. Argyros, R. Behl, S. S. Motsa. Ball Convergence for a Family of Quadrature-Based Methods for Solving Equations in Banach Space. Inter. J. Comput. Methods (2016): 1750017.

[14] P.K. Parida, D.K. Gupta. Recurrence relations for a Newton-like method in Banach spaces. J. Comput. Appl. Math. 206 (2007): 873-887.

[15] A. Cordero, J. R. Torregrosa. Variants of Newtons method using fifth-order quadrature formulas. Appl. Math. Comput. 190(2007): 686-698.

[16] S. Weerakoon, T. G. I. Fernando. A variant of Newton's method with accelerated third-order convergence. Appl. Math. Lett., 13 (2000): 87-93.

[17] M. A. Hernández-Verón, E. Martínez, Carles Teruel. Semilocal convergence of a k-step iterative process and its application for solving a special kind of conservative problems: DOI 10.1007/s11075016-0255-z.

[18] L.V. Kantorovich, G. P. Akilov. Functional Analysis, Pergamon Press, Oxford (1982).

[19] J.M. Ortega, W.C. Rheinboldt. Iterative solution of Nonlinear Equations in Several Variables. Academic, New York (1987).

[20] P. J. Davis, P. Rabinowitz. Methods of numerical integration. Courier Corporation, 2007. 